Comment. Math. Helv. 72 (1997) 128-166

(C) 1997 Birkhäuser Verlag, Basel

0010-2571/97/010128-39 \$1.50+0.20/0

Commentarii Mathematici Helvetici

\title{
Total positivity in Schubert varieties
}

\author{
Arkady Berenstein and Andrei Zelevinsky ${ }^{1}$
}

\begin{abstract}
We extend the results of [2] on totally positive matrices to totally positive elements in arbitrary semisimple groups.
\end{abstract}

Mathematics Subject Classification (1991). 14M15, 22E46, 15A48.

Keywords. Total positivity, semisimple Lie groups, Schubert varieties, Bruhat decomposition.

\section{Introduction}

In this paper we study the varieties of totally positive elements in Schubert cells for an arbitrary semisimple complex Lie group $G$. These varieties were introduced and studied by G. Lusztig in [10], [11]. Interest in them is motivated by a remarkable parallelism between their parametrizations and combinatorial labelings of the canonical basis for the quantum group corresponding to the maximal unipotent subgroup $N$ of $G$. In [2] these parametrizations were described quite explicitly for the type $A_{r}$, with the help of a special combinatorial substitution that we called the Chamber Ansatz. In the present paper, we generalize the results of [2] to arbitrary semisimple groups. In fact, practically all the results below can be extended in a straightforward way to arbitrary Kac-Moody algebras. For the sake of simplicity, we will not pursue this generalization here.

Now let us give a more systematic account of our main results. Let $\mathbf{g}$ be a semisimple complex Lie algebra of rank $r$ with the Cartan decomposition $\mathbf{g}=$ $\mathbf{n}_{-} \oplus \mathbf{h} \oplus \mathbf{n}$. Let $e_{i}, h_{i}, f_{i}(i=1, \ldots, r)$ be the standard generators of $\mathbf{g}$, and $A=\left(a_{i j}\right)$ be the Cartan matrix. The well-known commutation relations between the generators can be summarized as follows:

the Cartan subalgebra $\mathbf{h}$ is abelian with the basis $h_{1}, \ldots, h_{r}$;

the Lie algebra $\mathbf{n}$ (resp. $\mathbf{n}_{-}$) is generated by $e_{1}, \ldots, e_{r}$

\footnotetext{
${ }^{1}$ Partially supported by the NSF grant.
} 
(resp. by $f_{1}, \ldots, f_{r}$ ) subject to the Serre relations

$$
\begin{aligned}
& \left(\operatorname{ad} e_{i}\right)^{1-a_{i j}} e_{j}=0\left(\operatorname{resp} .\left(\operatorname{ad} f_{i}\right)^{1-a_{i j}} f_{j}=0\right) \text { for } i \neq j \\
& {\left[h_{i}, e_{j}\right]=a_{i j} e_{j},\left[h_{i}, f_{j}\right]=-a_{i j} f_{j},\left[e_{i}, f_{j}\right]=\delta_{i j} h_{i} .}
\end{aligned}
$$

Let $G$ be a simply connected complex Lie group with the Lie algebra $\mathbf{g}$. Let $N_{-}$, $H$ and $N$ be closed subgroups of $G$ with Lie algebras $\mathbf{n}_{-}, \mathbf{h}$ and $\mathbf{n}$, respectively; thus, $H$ is a maximal torus, and $N$ and $N_{-}$are two opposite maximal unipotent subgroups of $G$. Let $B_{-}=H N_{-}$and $B=H N$ be the corresponding pair of opposite Borel subgroups.

The Weyl group $W$ of $G$ is defined as $W=\operatorname{Norm}_{G}(H) / H$. For $w \in W$, we denote by $\bar{w}$ any representative of $w$ in the normalizer $\operatorname{Norm}_{G}(H)$. The action of $W$ on $H$ by conjugation gives rise to an action of $W$ on $\mathbf{h}$ and the dual action on $\mathbf{h}^{*}$. As usual, we will identify $W$ with the corresponding group of linear transformations of $\mathbf{h}^{*}$. The group $W$ is a Coxeter group generated by simple reflections $s_{1}, \ldots, s_{r}$ given by

$$
s_{i}(\gamma)=\gamma-\gamma\left(h_{i}\right) \alpha_{i}\left(\gamma \in \mathbf{h}^{*}\right)
$$

here $\alpha_{1}, \ldots, \alpha_{r} \in \mathbf{h}^{*}$ are simple roots given by $\alpha_{j}\left(h_{i}\right)=a_{i j}$. A reduced expression for $w \in W$ is a sequence of indices $\mathbf{i}=\left(i_{1}, \ldots, i_{m}\right)$ such that $w=s_{i_{1}} \cdots s_{i_{m}}$, and $m$ is the smallest length of such a factorization. We denote by $R(w)$ the set of reduced expressions for $w$. The length of any $\mathbf{i} \in R(w)$ is denoted by $l(w)$ and called the length of $w$.

For each $i=1, \ldots, r$, let $x_{i}(t)=\exp \left(t e_{i}\right)$ be a one-parameter subgroup in $N$ generated by $e_{i}$. Following G. Lusztig [10], we define the variety $N_{>0}$ of totally non-negative elements in $N$ as the multiplicative semigroup generated by all $x_{i}(t)$ with $i=1, \ldots, r$ and $t \geq 0$. For any $w \in W$, we set $N_{>0}^{w}=N_{\geq 0} \cap B_{-} w B_{-}$, the intersection of $N_{\geq 0}$ with the Bruhat cell corresponding to $w$. In view of the Bruhat decomposition $G=\bigcup_{w \in W} B_{-} w B_{-}$, the variety $N_{\geq 0}$ is a disjoint union of the $N_{>0}^{w}$ over all $w \in W$. The varieties $N_{>0}^{w}$ will be the main object of study in this paper.

We will think of $N$ as an open chart in the flag variety $B_{-} \backslash G$ of right cosets of $G$ modulo $B_{-}$(thus, $G$ acts on $B_{-} \backslash G$ from the right). To be more precise, we identify $N$ with its image under the natural projection $\pi: G \rightarrow B_{-} \backslash G$. Using this identification, each $N_{>0}^{w}$ becomes a subset of the corresponding Schubert cell $C_{w}=B_{-} \backslash B_{-} w B_{-}$.

For any sequence of indices $\mathbf{i}=\left(i_{1}, \ldots, i_{m}\right)$, we define the map $x_{\mathbf{i}}: \mathbf{C}^{m} \rightarrow N$ by

$$
x_{\mathbf{i}}\left(t_{1}, \ldots, t_{m}\right)=x_{i_{1}}\left(t_{1}\right) \cdots x_{i_{m}}\left(t_{m}\right) .
$$

The following proposition is due to Lusztig [10]; it shows that every $\mathbf{i} \in R(w)$ gives rise to a parametrization of elements of $N_{>0}^{w}$ by $m$-tuples of positive real numbers.

Proposition 1.1. Let $w \in W$, and $\mathbf{i}=\left(i_{1}, \ldots, i_{m}\right) \in R(w)$ be a reduced expression of $w$. Then the restriction of $x_{\mathbf{i}}$ to the set $\mathbf{R}_{>0}^{m}$ of $m$-tuples of positive real numbers is a bijection between $\mathbf{R}_{>0}^{m}$ and $N_{>0}^{w}$. 
Our first main result is an explicit formula for the inverse bijection $x_{\mathbf{i}}^{-1}: N_{>0}^{w} \rightarrow$ $\mathbf{R}_{>0}^{m}$. (In fact, $x_{\mathbf{i}}$ gives a birational isomorphism between $\mathbf{C}^{m}$ and the Schubert variety $X_{w}$, the closure of $C_{w}$ in $B_{-} \backslash G$; we compute the inverse isomorphism $x_{\mathbf{i}}^{-1}: X_{w} \rightarrow \mathbf{C}^{m}$.)

To formulate the answer, we need two ingredients. The first is a birational automorphism $\eta_{w}: X_{w} \rightarrow X_{w}$ defined as follows. Let $x \mapsto x^{T}$ be an involutive Lie algebra anti-automorphism of $\mathbf{g}$ given by

$$
e_{i}^{T}=f_{i}, f_{i}^{T}=e_{i}, h_{i}^{T}=h_{i}(i=1, \ldots, r)
$$

we will use the same notation $x \mapsto x^{T}$ for the corresponding involutive antiautomorphism of the group $G$. We set $N^{w}=N \cap B_{-} w B_{-}$; under the identification of $N$ with $\pi(N)$, the set $N^{w}$ becomes an open subset of the Schubert cell $C_{w}$.

Theorem 1.2. For every $z \in N^{w}$, the intersection $N \cap B_{-} w z^{T}$ consists of a unique element $\eta_{w}(z)$. The correspondence $z \mapsto \eta_{w}(z)$ is a regular automorphism of $N^{w}$. Furthermore, the restriction of $\eta_{w}$ to $N_{>0}^{w}$ is a bijection of $N_{>0}^{w}$ with itself.

The inverse map $\eta_{w}^{-1}: N^{w} \rightarrow N^{w}$ can be described as follows. Let $x \mapsto x^{\iota}$ be an involutive Lie algebra anti-automorphism of $\mathbf{g}$ given by

$$
e_{i}^{\iota}=e_{i}, f_{i}^{\iota}=f_{i}, h_{i}^{\iota}=-h_{i}(i=1, \ldots, r)
$$

we will use the same notation $x \mapsto x^{\iota}$ for the corresponding involutive antiautomorphism of the group $G$.

\section{Proposition 1.3.}

(a) For any $w \in W$, the map $x \mapsto x^{\iota}$ restricts to an isomorphism $N^{w} \rightarrow N^{w^{-1}}$ and to a bijection $N_{>0}^{w} \rightarrow N_{>0}^{w^{-1}}$.

(b) The map $\eta_{w}^{-1}: N^{w} \rightarrow N^{w}$ is given by

$$
\eta_{w}^{-1}(x)=\left(\eta_{w^{-1}}\left(x^{\iota}\right)\right)^{\iota}
$$

Theorem 1.2 and Proposition 1.3 will be proved in Section 5. In a special case when $G=S L_{r+1}$ is of the type $A_{r}$, and $w=w_{0}$ is the longest element of the Weyl group $W$ (which in this case is the symmetric group $S_{r+1}$ ), these results were obtained in [2], Lemma 1.3 and Theorem 3.2.5.

The second ingredient in our formula for $x_{\mathbf{i}}^{-1}$ is a family of regular functions $\Delta^{\gamma} \in \mathbf{C}[G]$, where $\gamma$ runs over extremal weights in the fundamental representations of $G$. To be more precise, we recall that the fundamental weights $\omega_{1}, \ldots, \omega_{r}$ form a basis in $\mathbf{h}^{*}$ dual to the basis $h_{1}, \ldots, h_{r}$ in $\mathbf{h}$. The free abelian subgroup $P \subset \mathbf{h}^{*}$ generated by the fundamental weights is the weight lattice of $G$. Every weight 
$\gamma \in P$ gives rise to a multiplicative character of the maximal torus $H$ which will be written as $d \mapsto d^{\gamma}(d \in H)$. We will call a weight $\gamma$ of the form $\gamma=w \omega_{i}$ for some $w \in W$ and $i=1, \ldots, r$ a chamber weight of level $i$. (This terminology is motivated by [2], where these weights in the $A_{r}$ case were associated with chambers in certain pseudo-line arrangements; in Section 7 we will extend this graphical interpretation of chamber weights to the types $B_{r}$ and $C_{r}$.) To every chamber weight $\gamma$ of level $i$ we associate a regular function $\Delta^{\gamma}$ on $G$, which is uniqely up to a scalar multiple determined by the condition that

$$
\Delta^{\gamma}\left(y d_{1} g d_{2}\right)=d_{1}^{\omega_{i}} d_{2}^{\gamma} \Delta^{\gamma}(g)\left(y \in N_{-}, g \in G, d_{1}, d_{2} \in H\right)
$$

(for more details and for the choice of a normalization of $\Delta^{\gamma}$ see Section 6 below). For example, if $G=S L_{r+1}$ then the chamber weights are in a natural correspondence with subsets $J \subset\{1, \ldots, r+1\}$, and the functions $\Delta^{\gamma}$ just introduced are the minors $\Delta^{J}$, where $\Delta^{J}(g)$ is the minor of $g$ with the row set $\{1,2, \ldots,|J|\}$ and the column set $J$.

Now we are in a position to formulate our main result.

Theorem 1.4. Suppose $\mathbf{i}=\left(i_{1}, \ldots, i_{m}\right) \in R(w)$, and $x=x_{\mathbf{i}}\left(t_{1}, \ldots, t_{m}\right) \in N_{>0}^{w}$. Then the components $t_{1}, \ldots, t_{m}$ are given by

$$
t_{k}=\frac{1}{\Delta^{w_{k} \omega_{i_{k}}}(z) \Delta^{w_{k+1} \omega_{i_{k}}}(z)} \prod_{j \neq i_{k}} \Delta^{w_{k} \omega_{j}}(z)^{-a_{j, i_{k}}},
$$

where $z=\eta_{w}^{-1}(x)$, and

$$
w_{k}=w_{k}^{\mathbf{i}}=s_{i_{m}} s_{i_{m-1}} \cdots s_{i_{k}}
$$

(with the convention $w_{m+1}=e$, the identity element of $W$ ).

This theorem will be proved in Section 6. When $G=S L_{r+1}$ and $w=w_{0}$, it specializes to Theorem 1.4 in [2]. Note that a generalization of this result to an arbitrary element $w$ of the Weyl group (for the type $A_{r}$ ) obtained in [2], Theorem 5.4.2, is different from the one given by Theorem 1.4 although closely related to it. In Section 6 we will also prove a version of Theorem 1.4 that generalizes [2], Theorem 5.4.2. An advantage of the present version is that it implies a family of criteria for total positivity that generalize those given by [2], Theorem 3.2.1 for $G=S L_{r+1}$ and $w=w_{0}$ (the possibility of extending these criteria to an arbitrary $w \in W$ was not realized in [2]). Each of these criteria says that a point $x \in N^{w}$ belongs to $N_{>0}^{w}$ if and only if a certain system of $m$ regular functions (where $m=l(w)=\operatorname{dim}\left(N^{w}\right)$ ) takes positive values on $x$. More precisely, we will show that Theorem 1.4 implies the following.

Theorem 1.5. Suppose $\mathbf{i}=\left(i_{1}, \ldots, i_{m}\right) \in R(w)$. A point $x \in N^{w}$ belongs to $N_{>0}^{w}$ if and only if $\Delta^{w_{k} \omega_{i_{k}}}(x)>0$ for $k=1, \ldots, m$, where $w_{k}=w_{k}^{\mathbf{i}}$ is given by (1.11). 
This theorem sharpens the following result by G. Lusztig: the variety $N_{\geq 0}$ consists of elements of $N$ that act on the canonical basis in every fundamental representation of $G$ by a matrix with non-negative entries, see [11].

Another application of Theorem 1.4 is an explicit formula for the transition maps that relate parametrizations of $N_{>0}^{w}$ associated to different reduced expressions of $w$. To be more precise, for every two reduced expressions $\mathbf{i}, \mathbf{i}^{\prime} \in R(w)$ the transition map $R_{\mathbf{i}}^{\mathbf{i}^{\prime}}: \mathbf{R}_{>0}^{m} \rightarrow \mathbf{R}_{>0}^{m}$ is defined by

$$
R_{\mathbf{i}}^{\mathbf{i}^{\prime}}=x_{\mathbf{i}^{\prime}}^{-1} \circ x_{\mathbf{i}}
$$

An explicit formula for $R_{\mathbf{i}}^{\mathbf{i}^{\prime}}$ can be obtained by simply combining formula (1.5) for $x_{\mathbf{i}}$ and (1.10) for $x_{\mathbf{i}^{\prime}}^{-1}$.

In Section 3 we will prove that transition maps have the following positivity property.

Theorem 1.6. The components of the vector $t^{\prime}=R_{\mathbf{i}}^{\mathbf{i}^{\prime}}(t)$ are subtraction-free rational expressions in the components of $t$.

The proof is based on the following well-known property of reduced expressions in Coxeter groups (see [3], [6]).

Proposition 1.7. Every two reduced expressions of the same element $w \in W$ can be obtained from each other by a sequence of d-moves.

Here a $d$-move is the following operation on reduced expressions: replacing $d$ consecutive entries $i, j, i, j, \ldots$ by $j, i, j, i, \ldots$, where $d$ is the order of $s_{i} s_{j}$ in $W$. The value of $d$ is determined as follows:

if $a_{i j} a_{j i}=0$ (resp. 1,2 , or 3$)$ then the order $d$ of $s_{i} s_{j}$ is 2 (resp. 3,4 , or 6$)$.

Proposition 1.7 reduces the proof of Theorem 1.6 to its special case when the reduced expressions $\mathbf{i}$ and $\mathbf{i}^{\prime}$ have the form $\mathbf{i}=(i, j, i, \ldots), \mathbf{i}^{\prime}=(j, i, j, \ldots)$, with the length $d$ of both sequences given by (1.13). For $d=2$ and $d=3$, the transition $\operatorname{map} R_{\mathbf{i}}^{\mathbf{i}^{\prime}}$ was computed by G. Lusztig (see [9] or [2], (1.13) and (1.14)); this proves Theorem 1.6 in a simply-laced case. In Section 3 we compute $R_{\mathbf{i}}^{\mathbf{i}^{\prime}}$ in the two remaining cases $d=4$ and $d=6$.

As in [2], the fact that transition maps are subtraction-free opens up an opportunity to define them over an arbitrary semifield $K$ instead of $\mathbf{R}_{>0}$ (see [2], Section 2 or Section 4 below for the precise definition of what we mean by a semifield). Following [2], we then introduce the Lusztig variety $\mathcal{L}^{w}=\mathcal{L}^{w}(K)$. An element $\mathbf{t}$ of $\mathcal{L}^{w}$ is, by definition, a tuple

$$
\mathbf{t}=\left(t^{\mathbf{i}}\right) \mathbf{i} \in R(w)
$$


where each $t^{\mathbf{i}}=\left(t_{1}^{\mathbf{i}}, \ldots, t_{m}^{\mathbf{i}}\right)$ is a "vector" in $K^{m}$, and these vectors satisfy the relations $t^{\mathbf{i}^{\prime}}=R_{\mathbf{i}}^{\mathbf{i}^{\prime}}\left(t^{\mathbf{i}}\right)$ for all $\mathbf{i}, \mathbf{i}^{\prime} \in R(w)$. The Lusztig variety is studied in Section 4 below. We develop an "abstract version" of the Chamber Ansatz (1.10), where the functions $\Delta^{\gamma}(z)$ are replaced by variables $M_{\gamma}$ taking values in the ground semifield $K$. The formulas for the transition maps associated with $d$-moves then translate into (subtraction-free) polynomial relations between the $M_{\gamma}$. For $d=3$ these relations essentially coincide with the quadratic 3 -term relations obtained in [2], (2.5.4). In Section 4 we find these relations for $d=4$ and $d=6$ : it turns out that each 4 -move produces two relations of degrees 3 and 4 , while a 6 -move produces four relations of degrees $6,9,10$, and 15. In view of Theorem 1.4, the functions $\Delta^{\gamma}$ satisfy all these relations in the coordinate ring $\mathbf{C}[G]$.

An especially interesting choice of the ground semifield $K$ is $K=\mathbf{Z}$, where the usual addition plays the role of multiplication, and taking the minimum plays the role of addition (this is the so-called tropical semifield which played an important part in [2]). Over this semifield, the transition maps become certain piecewiselinear transformations. When $G$ is simply-laced, and $w=w_{0}$, the Lusztig variety $\mathcal{L}^{w}$ over the tropical semifield is naturally identified with the canonical basis for the quantum group corresponding to $N$ (this observation due to Lusztig was a main motivation for our interest in total positivity). In a non-simply laced case, or when $w \neq w_{0}$, the relationship between the totally positive varieties and the canonical basis is much less understood. However, one can show that for the type $B_{2}$, the piecewise-linear version of our formula for the transition map (see (3.4) below) is equivalent to the formula for the canonical basis given in [8], Section 12.5 (we thank the anonymous referee for pointing this out to us).

We would like to note once again that the above results can be extended to arbitrary Cartan matrices and corresponding Kac-Moody algebras. It is also possible to "quantize" the maps $x_{\mathbf{i}}$ by constructing their $q$-deformations. Some results in this direction were obtained in [1]; in fact, most of the results in the present paper have their "quantum analogues". They will be discussed in a separate publication.

The paper is organized as follows. In Section 2 we discuss the properties of chamber weights. In Section 3, we compute the transition maps corresponding to $d$-moves; as explained above, the fact that the answers are subtraction-free implies Theorem 1.6. An "abstract" version of the Chamber Ansatz is introduced and studied in Section 4. Theorem 1.2 and Proposition 1.3 are proved in Section 5, while Theorems 1.4 and 1.5 are proved in Section 6. Finally, in Section 7 we give a graphical interpretation of our general results for the types $A_{r}, B_{r}$, and $C_{r}$.

ACKNOWLEDGMENTs. This work was partly done during the visit of one of the authors (A.Z.) to the University of Basel, Switzerland, in May-June 1996. He is grateful to Hanspeter Kraft for his hospitality. 


\section{Chamber weights}

In this section we develop the properties of chamber weights. For the convenience of the reader, we start by recalling some well known results about roots, weights, reduced expressions, etc. We retain the notation of Section 1. Thus, $\mathbf{g}$ is a semisimple complex Lie algebra of rank $r$ with the Cartan decomposition $\mathbf{g}=\mathbf{n}_{-} \oplus \mathbf{h} \oplus \mathbf{n}$ satisfying the properties (1.1) through (1.3). The root lattice $Q$ is a free abelian subgroup in $\mathbf{h}^{*}$ generated by simple roots $\alpha_{1}, \ldots, \alpha_{r}$. The root decomposition of $\mathbf{g}$ is written as $\mathbf{g}=\mathbf{h} \oplus \bigoplus_{\alpha \in \Phi} \mathbf{g}(\alpha)$, where $\Phi$ is the root system of $\mathbf{g}$. Let $Q_{+}$be the additive semigroup in $Q$ generated by $\alpha_{1}, \ldots, \alpha_{r}$. Then $\Phi$ is the disjoint union $\Phi_{+} \cup\left(-\Phi_{+}\right)$, where $\Phi_{+}=\Phi \cap Q_{+}$is the set of positive roots; thus, $\mathbf{n}=\bigoplus_{\alpha \in \Phi_{+}} \mathbf{g}(\alpha)$ and $\mathbf{n}_{-}=\bigoplus_{\alpha \in \Phi_{+}} \mathbf{g}(-\alpha)$.

The Cartan matrix $A$ of $\mathbf{g}$ has the following well-known properties:

$$
\begin{aligned}
a_{i i}=2 \text { for } & i=1, \ldots, r, \text { and } a_{i j} \in\{0,-1,-2,-3\} \text { for } i \neq j ; \\
& \text { the matrix } C=\left(c_{i j}=d_{i} a_{i j}\right) \text { is symmetric } \\
& \text { for some positive integers } d_{1}, \ldots, d_{r} .
\end{aligned}
$$

We fix the matrix $C$ as in (2.2), and define a symmetric bilinear form on $\mathbf{h}^{*}$ by

$$
\left(\alpha_{i}, \alpha_{j}\right)=c_{i j}=d_{i} a_{i j} .
$$

This form makes the real vector space $\mathbf{h}_{\mathbf{R}}^{*}$ generated by the simple roots into an Euclidean space. Each simple reflection $s_{i} \in W$ acts on this space as an orthogonal reflection:

$$
s_{i}(\gamma)=\gamma-\left(\gamma, \alpha_{i}^{\vee}\right) \alpha_{i}
$$

where $\alpha^{\vee}=2 \alpha /(\alpha, \alpha)$. In particular, the action of $s_{i}$ on the simple roots $\alpha_{j}$, simple coroots $\alpha_{j}^{\vee}$ and fundamental weights $\omega_{j}$ is given by

$$
s_{i}\left(\alpha_{j}\right)=\alpha_{j}-a_{i j} \alpha_{i}, s_{i}\left(\alpha_{j}^{\vee}\right)=\alpha_{j}^{\vee}-a_{j i} \alpha_{i}^{\vee}, s_{i}\left(\omega_{j}\right)=\omega_{j}-\delta_{i j} \alpha_{i} .
$$

It also follows that the scalar product is $W$-invariant.

For an element $w \in W$ we define its inversion set by

$$
\operatorname{Inv}(w)=\Phi_{+} \cap w^{-1}\left(-\Phi_{+}\right) .
$$

It is known that the cardinality of $\operatorname{Inv}(w)$ is equal to $l(w)$. Furthermore, $\operatorname{Inv}(w)$ can be recovered from any reduced expression $\mathbf{i}=\left(i_{1}, \ldots, i_{m}\right) \in R(w)$ as follows. As in (1.11) above, we set

$$
w_{k}^{\mathbf{i}}=s_{i_{m}} s_{i_{m-1}} \cdots s_{i_{k}}
$$

(with the convention $w_{m+1}^{\mathbf{i}}=e$, the identity element of $W$ ). We also set

$$
\alpha_{k}^{\mathbf{i}}=w_{k+1}^{\mathbf{i}}\left(\alpha_{i_{k}}\right) .
$$

The following proposition is well known, see [3], VI,1.6. 
Proposition 2.1. The roots $\alpha_{k}^{\mathbf{i}}$ for $k=1, \ldots, m$ are distinct, and $\left\{\alpha_{1}^{\mathbf{i}}, \alpha_{2}^{\mathbf{i}}, \ldots\right.$, $\left.\alpha_{m}^{\mathbf{i}}\right\}=\operatorname{Inv}(w)$.

This proposition has several important corollaries. For the convenience of the reader, we provide the proofs of those of them that seem to be less well known.

Corollary 2.2. A simple root $\alpha_{i}$ belongs to $\operatorname{Inv}(w)$ if and only if $l\left(w s_{i}\right)=l(w)-$ 1 ; in this case we have $\operatorname{Inv}(w)=\left\{\alpha_{i}\right\} \cup s_{i}\left(\operatorname{Inv}\left(w s_{i}\right)\right)$. Similarly, $\alpha_{i}$ belongs to $\operatorname{Inv}\left(w^{-1}\right)$ if and only if $l\left(s_{i} w\right)=l(w)-1$; in this case we have $\operatorname{Inv}(w)=$ $\left\{-w^{-1}\left(\alpha_{i}\right)\right\} \cup \operatorname{Inv}\left(s_{i} w\right)$.

Corollary 2.3. The following two conditions on a pair of elements $u, w \in W$ are equivalent:

(a) $l(w)=l(u)+l\left(w u^{-1}\right)$;

(b) $\operatorname{Inv}(u) \subset \operatorname{Inv}(w)$.

Proof. The implication (a) $\Rightarrow$ (b) follows from the second statement in Corollary 2.2. The reverse implication (b) $\Rightarrow(\mathrm{a})$ is trivial if $u=e$, so we can assume that $u \neq$ $e$, i.e., $\operatorname{Inv}(u) \neq \emptyset$. Let $\alpha_{i} \in \operatorname{Inv}(u) \subset \operatorname{Inv}(w)$. Using the first statement in Corollary 2.2, we conclude that $l\left(w s_{i}\right)=l(w)-1, l\left(u s_{i}\right)=l(u)-1$, and $\operatorname{Inv}\left(u s_{i}\right) \subset$ Inv $\left(w s_{i}\right)$. Using induction on $l(u)$ we can assume that (a) is true when $w$ and $u$ are replaced with $w s_{i}$ and $u s_{i}$, respectively. Clearly, this implies that (a) is also true for $w$ and $u$, and we are done.

Corollary 2.4. For $i=1, \ldots, r$ and $w \in W$, we have $w\left(\omega_{i}\right) \in \omega_{i}-Q_{+}$. Furthermore, the stabilizer of $\omega_{i}$ in $W$ is the subgroup $W_{\widehat{i}} \subset W$ generated by $\left\{s_{j}: j \neq i\right\}$.

Corollary 2.5. Each coset in $W / W_{\widehat{i}}$ has the unique representative of minimal length. Furthermore, $u \in W$ is the element of minimal length in its coset $u W_{\hat{i}}$ if and only if $l\left(u s_{j}\right)=l(u)+1$ for all $j \neq i$; in this case $l(u v)=l(u)+l(v)$ for all $v \in W_{\widehat{i}}$.

Let us now apply the above results to the study of chamber weights. Recall from the introduction that by a chamber weight of level $i$ we mean a weight in $W \omega_{i}$, the $W$-orbit of the $i$-th fundamental weight (in view of Corollary 2.4, the orbits $W \omega_{i}$ are disjoint, so the level is well-defined). Every such weight $\gamma$ can be uniquely written as $\gamma=u \omega_{i}$, where $u$ is of minimal length in its coset $u W_{\widehat{i}}$; this presentation will be called the minimal presentation of $\gamma$. We set

$$
I(\gamma)=\left\{\alpha \in \Phi_{+}:\left(\gamma, \alpha^{\vee}\right)<0\right\} .
$$

Proposition 2.6. If $\gamma=w \omega_{i}$ for some $w \in W$ then $I(\gamma) \subset \operatorname{Inv}\left(w^{-1}\right)$, and $\left(\gamma, \alpha^{\vee}\right)=0$ for all $\alpha \in \operatorname{Inv}\left(w^{-1}\right)-I(\gamma)$. A presentation $\gamma=u \omega_{i}$ is minimal if and only if $I(\gamma)=\operatorname{Inv}\left(u^{-1}\right)$. 
Proof. Let $\gamma=w \omega_{i}$. If $\alpha \in I(\gamma)$ then $0>\left(\gamma, \alpha^{\vee}\right)=\left(\omega_{i}, w^{-1} \alpha^{\vee}\right)$, hence $w^{-1} \alpha \in$ $-\Phi_{+}$, i.e., $\alpha \in \operatorname{Inv}\left(w^{-1}\right)$. This proves the inclusion $I(\gamma) \subset \operatorname{Inv}\left(w^{-1}\right)$. Moreover, the same equality $\left(\gamma, \alpha^{\vee}\right)=\left(\omega_{i}, w^{-1} \alpha^{\vee}\right)$ shows that $\left(\gamma, \alpha^{\vee}\right) \leq 0$ for all $\alpha \in$ $\operatorname{Inv}\left(w^{-1}\right)$; therefore, $\left(\gamma, \alpha^{\vee}\right)=0$ for all $\alpha \in \operatorname{Inv}\left(w^{-1}\right)-I(\gamma)$.

It remains to prove the inclusion $\operatorname{Inv}\left(u^{-1}\right) \subset I(\gamma)$ for the minimal presentation $\gamma=u \omega_{i}$. We proceed by induction on $l(u)$. There is nothing to prove in the case when $l(u)=0$, so we can assume that $u=s_{j} u^{\prime}$ for some $j$, where $l(u)=l\left(u^{\prime}\right)+1$. Let $\gamma^{\prime}=s_{j} \gamma$; it is easy to see that $\gamma^{\prime}$ has the minimal presentation $\gamma^{\prime}=u^{\prime} \omega_{i}$. By induction, we can assume that $\left(\gamma^{\prime}, \alpha^{\prime \vee}\right)<0$ for all $\alpha^{\prime} \in \operatorname{Inv}\left(u^{\prime-1}\right)$. On the other hand, we have $\operatorname{Inv}\left(u^{-1}\right)=\left\{\alpha_{j}\right\} \cup s_{j}\left(\operatorname{Inv}\left(u^{\prime-1}\right)\right)$, in view of Corollary 2.2. If $\alpha=s_{j} \alpha^{\prime} \in s_{j}\left(\operatorname{Inv}\left(u^{\prime-1}\right)\right)$ then $\left(\gamma, \alpha^{\vee}\right)=\left(\gamma^{\prime}, \alpha^{\prime \vee}\right)<0$. To complete the proof, it remains to show that $\left(\gamma, \alpha_{j}^{\vee}\right) \neq 0$, or, equivalently, that $s_{j} \gamma \neq \gamma$. But the last inequality follows from the fact that $\gamma=u \omega_{i}$ is the minimal presentation of $\gamma$. This completes the proof of Proposition 2.6.

The minimal presentation of a chamber weight can be extracted from an arbitrary presentation as follows.

Proposition 2.7. Let $\gamma=w \omega_{i}$ be a chamber weight of level $i$, and let $\mathbf{i}=$ $\left(i_{1}, \ldots, i_{m}\right) \in R(w)$. The minimal presentation of $\gamma$ is obtained from the expression $\gamma=s_{i_{1}} \cdots s_{i_{m}} \omega_{i}$ by removing all the factors $s_{i_{k}}$ for which $\left(\omega_{i},\left(\alpha_{k}^{\mathbf{i}}\right)^{\vee}\right)=0$.

Proof. Let $u$ be the element obtained from the product $w=s_{i_{1}} \cdots s_{i_{m}}$ by removing all the factors $s_{i_{k}}$ for which $\left(\omega_{i},\left(\alpha_{k}^{\mathbf{i}}\right)^{\vee}\right)=0$. Using $(2.4)$, it is easy to show that the equality $\left(\omega_{i},\left(\alpha_{k}^{\mathbf{i}}\right)^{\vee}\right)=0$ is equivalent to

$$
s_{i_{k}} \cdots s_{i_{m}} \omega_{i}=s_{i_{k+1}} \cdots s_{i_{m}} \omega_{i}
$$

It is now obvious that $u \omega_{i}=w \omega_{i}$, so it remains to show that the presentation $\gamma=u \omega_{i}$ is minimal. Rewriting the scalar product $\left(\omega_{i},\left(\alpha_{k}^{\mathbf{i}}\right)^{\vee}\right)$ as $\left(\gamma, w\left(\alpha_{k}^{\mathbf{i}}\right)^{\vee}\right)$ and using Proposition 2.6, we conclude that the equality $\left(\omega_{i},\left(\alpha_{k}^{\mathbf{i}}\right)^{\vee}\right)=0$ is also equivalent to the condition that $-w\left(\alpha_{k}^{\mathbf{i}}\right)^{\vee} \in \operatorname{Inv}\left(w^{-1}\right)-I(\gamma)$. Removing the corresponding terms $s_{i_{k}}$ from the reduced factorization of $w$, we express $u$ as the product of $|I(\gamma)|$ simple reflections. Now the minimality of the presentation $\gamma=u \omega_{i}$ follows from the last statement in Proposition 2.6, and we are done.

Let $\mathbf{i}=\left(i_{1}, \ldots, i_{m}\right) \in R(w)$ for some $w \in W$. We say that a chamber weight $\gamma$ is an $\mathbf{i}$-chamber weight if $\gamma=w_{k}^{\mathbf{i}} \omega_{i}$ for some $k=1, \ldots, m+1$ and $i=1, \ldots, r$ (see $(2.7))$. Let $E^{\mathbf{i}}$ denote the set of all $\mathbf{i}$-chamber weights, and let $E^{w}=\cup_{\mathbf{i} \in R(w)} E^{\mathbf{i}}$. The elements of $E^{w}$ will be called w-chamber weights. The following characterization of $w$-chamber weights generalizes [2], Proposition 5.3.1.

Proposition 2.8. A chamber weight $\gamma$ is a w-chamber weight if and only if $I(\gamma) \subset$ $\operatorname{Inv}(w)$. 
Proof. Let $\gamma=u \omega_{i}$ be the minimal presentation of a chamber weight $\gamma$. In view of Corollary 2.3 and Proposition 2.6, the inclusion $I(\gamma) \subset \operatorname{Inv}(w)$ is equivalent to $l(w)=l\left(u^{-1}\right)+l(w u)$. Clearly, the last condition implies that $u=w_{k}^{\mathbf{i}}$ for some $\mathbf{i} \in R(w)$ and $k=1, \ldots, m+1$, hence that $\gamma$ is a $w$-chamber weight. Conversely, if $\gamma=w_{k}^{\mathbf{i}} \omega_{i}$ is a $w$-chamber weight then $w_{k}^{\mathbf{i}} \in u W_{\widehat{i}}$, so Corollaries 2.3 and 2.5 and Proposition 2.6 imply that $I(\gamma)=\operatorname{Inv}\left(u^{-1}\right) \subset \operatorname{Inv}\left(\left(w_{k}^{\mathbf{i}}\right)^{-1}\right) \subset \operatorname{Inv}(w)$, as desired.

We now turn to the study of $\mathbf{i}$-chamber weights, where $\mathbf{i}=\left(i_{1}, \ldots, i_{m}\right) \in R(w)$ is a fixed reduced expression. For $k=1, \ldots, m$, we set

$$
\gamma(k ; \mathbf{i})=w_{k}^{\mathbf{i}} \omega_{i_{k}} .
$$

Proposition 2.9. The set $E^{\mathbf{i}}$ consists of $m+r$ elements: the $\gamma(k ; \mathbf{i})$ for $k=$ $1, \ldots, m$, and the fundamental weights $\omega_{1}, \ldots, \omega_{r}$.

Proof. Let $\gamma=w_{k}^{\mathbf{i}} \omega_{i} \in E^{\mathbf{i}}$, where $1 \leq k \leq m+1$ and $1 \leq i \leq r$. If $i_{l} \neq i$ for $l \geq k$ (in particular, if $k=m+1$ ) then $\gamma=\omega_{i}$, in view of Corollary 2.4. Otherwise, let $l$ be the minimal index such that $l \geq k$ and $i_{l}=i$. Again using Corollary 2.4, we see that $\gamma=\gamma(l ; \mathbf{i})$. The fact that the elements $\gamma(k ; \mathbf{i})$ and $\omega_{i}$ are all distinct, is also an easy consequence of Corollary 2.4 .

In general, the presentation $(2.10)$ of $\gamma(k ; \mathbf{i})$ is not minimal. The minimal presentation can be extracted from it with the help of Proposition 2.7, which now takes the following form.

Corollary 2.10. The minimal presentation of $\gamma=\gamma(k ; \mathbf{i})$ is obtained from the presentation $\gamma=s_{i_{m}} \cdots s_{i_{k}} \omega_{i_{k}}$ by removing all the terms $s_{i_{l}}$ for which $\left(\gamma,\left(\alpha_{l}^{\mathbf{i}}\right)^{\vee}\right)=$ 0.

We conclude this section with the following proposition that generalizes Lemma 2.7.2 in [2].

Proposition 2.11. For any $w$-chamber weight $\gamma$, the set $R(w ; \gamma)=\{\mathbf{i} \in R(w)$ : $\left.\gamma \in E^{\mathbf{i}}\right\}$ is connected with respect to d-moves.

Proof. Recall that $d$-moves were defined in the introduction, after Proposition 1.7 (in fact, Proposition 1.7 is a special case of Proposition 2.11). Let $\mathbf{i} \in R(w ; \gamma)$, i.e., $\gamma=w_{k}^{\mathbf{i}} \omega_{i}$ for some $k=1, \ldots, m+1$ and $i=1, \ldots, r$. We will write $\mathbf{i}$ as a concatenation $\left(\mathbf{i}^{1}, \mathbf{i}^{2}\right)$, where $\mathbf{i}^{1}=\left(i_{1}, \ldots, i_{k-1}\right)$ and $\mathbf{i}^{2}=\left(i_{k}, \ldots, i_{m}\right)$. By Corollary 2.4, the index $i$ and the coset $w_{k}^{\mathbf{i}} W_{\widehat{i}} \in W / W_{\widehat{i}}$ depend only on $\gamma$. Let $u$ be the minimal length representative in $w_{k}^{i} W_{\widehat{i}}$, i.e, $\gamma=u \omega_{i}$ is the minimal presentation of $\gamma$. By Corollary 2.5, $l\left(w_{k}^{\mathbf{i}}\right)=l(u)+l\left(u^{-1} w_{k}^{\mathbf{i}}\right)$. It easily follows that $l(w)=l(w u)+l\left(u^{-1}\right)$. Let us fix some reduced expressions $\mathbf{j}^{1} \in R(w u)$ 
and $\mathbf{j}^{2} \in R\left(u^{-1}\right)$. It is enough to show that $\mathbf{i}$ can be transformed to $\left(\mathbf{j}^{1}, \mathbf{j}^{2}\right)$ by a sequence of $d$-moves staying at all times in $R(w ; \gamma)$. This can be done in two steps, each time using Proposition 1.7. First, we can transform $\mathbf{i}$ into a reduced expression of the form $\left(\mathbf{i}^{1}, \mathbf{i}^{\prime}, \mathbf{j}^{2}\right)$ by a sequence of $d$-moves applied to $\mathbf{i}^{2} \in R\left(\left(w_{k}^{\mathbf{i}}\right)^{-1}\right)$. Second, we can transform $\left(\mathbf{i}^{1}, \mathbf{i}^{\prime}, \mathbf{j}^{2}\right)$ to $\left(\mathbf{j}^{1}, \mathbf{j}^{2}\right)$ by a sequence of $d$ moves applied to $\left(\mathbf{i}^{1}, \mathbf{i}^{\prime}\right) \in R(w u)$. Clearly, all the intermediate reduced expressions in this process belong to $R(w ; \gamma)$. This completes the proof of Proposition 2.11.

\section{Transition maps}

In this section we study transition maps $R_{\mathbf{i}}^{\mathbf{i}^{\prime}}: \mathbf{R}_{>0}^{m} \rightarrow \mathbf{R}_{>0}^{m}$ (recall that they are defined by (1.12) in the introduction). We will find explicit formulas for $R_{\mathbf{i}}^{\mathbf{i}^{\prime}}$, where the reduced expressions $\mathbf{i}$ and $\mathbf{i}^{\prime}$ have the form $\mathbf{i}=(i, j, i, \ldots), \mathbf{i}^{\prime}=(j, i, j, \ldots)$, with the length $d$ of both sequences given by (1.13). We will use the notation $R_{i j i \ldots}^{j i j \ldots}\left(t_{1}, \ldots, t_{d}\right)=\left(p_{1}, \ldots, p_{d}\right)$; thus, the tuples $t_{1}, \ldots, t_{d}$ and $p_{1}, \ldots, p_{d}$ are related by

$$
x_{i}\left(t_{1}\right) x_{j}\left(t_{2}\right) x_{i}\left(t_{3}\right) \cdots=x_{j}\left(p_{1}\right) x_{i}\left(p_{2}\right) x_{j}\left(p_{3}\right) \cdots .
$$

If $a_{i j}=a_{j i}=0$, and so $d=2$ then $x_{i}(t)$ and $x_{j}(s)$ commute with each other for all $s$ and $t$, hence the transition map $R_{i j}^{j i}$ is given by

$$
p_{1}=t_{2}, p_{2}=t_{1} .
$$

The remaining three cases when $d=3,4$, or 6 are treated in the following theorem.

\section{Theorem 3.1.}

(a) Let $a_{i j}=a_{j i}=-1$, so $d=3$. The transition map $R_{i j i}^{j i j}$ is given by

$$
p_{1}=\frac{t_{2} t_{3}}{t_{1}+t_{3}}, p_{2}=t_{1}+t_{3}, p_{3}=\frac{t_{1} t_{2}}{t_{1}+t_{3}} .
$$

(b) Let $a_{i j}=-2, a_{j i}=-1$, so $d=4$. The transition map $R_{i j i j}^{j i j i}$ is given by

$$
p_{1}=\frac{t_{2} t_{3}^{2} t_{4}}{\pi_{2}}, p_{2}=\frac{\pi_{2}}{\pi_{1}}, p_{3}=\frac{\pi_{1}^{2}}{\pi_{2}}, p_{4}=\frac{t_{1} t_{2} t_{3}}{\pi_{1}}
$$

where

$$
\pi_{1}=t_{1} t_{2}+\left(t_{1}+t_{3}\right) t_{4}, \pi_{2}=t_{1}^{2} t_{2}+\left(t_{1}+t_{3}\right)^{2} t_{4} .
$$

(c) Let $a_{i j}=-3, a_{j i}=-1$, so $d=6$. The transition map $R_{i j i j i j}^{j i j i j i}$ is given by

$$
p_{1}=\frac{t_{2} t_{3}^{3} t_{4}^{2} t_{5}^{3} t_{6}}{\pi_{3}}, p_{2}=\frac{\pi_{3}}{\pi_{2}}, p_{3}=\frac{\pi_{2}^{3}}{\pi_{3} \pi_{4}}, p_{4}=\frac{\pi_{4}}{\pi_{1} \pi_{2}}, p_{5}=\frac{\pi_{1}^{3}}{\pi_{4}}, p_{6}=\frac{t_{1} t_{2} t_{3}^{2} t_{4} t_{5}}{\pi_{1}}
$$


where

$$
\begin{gathered}
\pi_{1}=t_{1} t_{2} t_{3}^{2} t_{4}+t_{1} t_{2}\left(t_{3}+t_{5}\right)^{2} t_{6}+\left(t_{1}+t_{3}\right) t_{4} t_{5}^{2} t_{6} \\
\pi_{2}=t_{1}^{2} t_{2}^{2} t_{3}^{3} t_{4}+t_{1}^{2} t_{2}^{2}\left(t_{3}+t_{5}\right)^{3} t_{6}+\left(t_{1}+t_{3}\right)^{2} t_{4}^{2} t_{5}^{3} t_{6}+t_{1} t_{2} t_{4} t_{5}^{2} t_{6}\left(3 t_{1} t_{3}+2 t_{3}^{2}+2 t_{3} t_{5}+2 t_{1} t_{5}\right) \\
\pi_{3}=t_{1}^{3} t_{2}^{2} t_{3}^{3} t_{4}+t_{1}^{3} t_{2}^{2}\left(t_{3}+t_{5}\right)^{3} t_{6}+\left(t_{1}+t_{3}\right)^{3} t_{4}^{2} t_{5}^{3} t_{6}+t_{1}^{2} t_{2} t_{4} t_{5}^{2} t_{6}\left(3 t_{1} t_{3}+3 t_{3}^{2}+3 t_{3} t_{5}+2 t_{1} t_{5}\right) \\
\pi_{4}=t_{1}^{2} t_{2}^{2} t_{3}^{3} t_{4}\left(t_{1} t_{2} t_{3}^{3} t_{4}+2 t_{1} t_{2}\left(t_{3}+t_{5}\right)^{3} t_{6}+\left(3 t_{1} t_{3}+3 t_{3}^{2}+3 t_{3} t_{5}+2 t_{1} t_{5}\right) t_{4} t_{5}^{2} t_{6}\right) \\
+t_{6}^{2}\left(t_{1} t_{2}\left(t_{3}+t_{5}\right)^{2}+\left(t_{1}+t_{3}\right) t_{4} t_{5}^{2}\right)^{3}
\end{gathered}
$$

Proof. One way to prove our theorem would be to compute both sides of (3.1) in some matrix representation of the group generated by one-parameter subgroups $x_{i}(t)$ and $x_{j}(s)$. For this purpose, one could use standard matrix representations of rank two semisimple groups of types $A_{2}, B_{2}$ (or $C_{2}$ ) and $G_{2}$. For the type $A_{2}$, this was done in [9]. Using a more uniform approach, we will realize the group $N$ as a multiplicative subgroup in the completion $\widehat{\mathcal{U}}$ of the universal enveloping algebra $\mathcal{U}=U(\mathbf{n})$ of $\mathbf{n}$. (This realization of $N$ makes sense for an arbitrary KacMoody algebra.) To be more precise, recall that $\mathcal{U}$ is an associative algebra with unit generated by $e_{1}, \ldots, e_{r}$ subject to the Serre relations $\left(\operatorname{ad} e_{i}\right)^{1-a_{i j}}\left(e_{j}\right)=0$ for $i \neq j$, where ad $x(y)=x y-y x$. The algebra $\mathcal{U}$ is $Q_{+}$-graded via $\operatorname{deg}\left(e_{i}\right)=\alpha_{i}$; the homogeneous component of degree $\gamma \in Q_{+}$in $\mathcal{U}$ will be denoted $\mathcal{U}(\gamma)$. The completion $\widehat{\mathcal{U}}$ consists of formal infinite sums $\sum_{\gamma \in Q_{+}} u_{\gamma}$, where $u_{\gamma} \in \mathcal{U}(\gamma)$. The multiplication

$$
\left(\sum_{\gamma} u_{\gamma}\right)\left(\sum_{\gamma} v_{\gamma}\right)=\sum_{\gamma}\left(\sum_{\gamma^{\prime}} u_{\gamma^{\prime}} v_{\gamma-\gamma^{\prime}}\right)
$$

makes $\widehat{\mathcal{U}}$ into an associative algebra. The group $N$ is embedded into the multiplicative group of $\widehat{\mathcal{U}}$ via

$$
x_{i}(t)=\exp \left(t e_{i}\right)=\sum_{n \geq 0} t^{n} e_{i}^{(n)}
$$

where the notation $e^{(n)}$ stands for the divided power $e^{n} / n$ !.

(a) To prove (3.3), we consider the basis $\left\{e_{i}, e_{j}, e_{i} e_{j}, e_{j} e_{i}\right\}$ in the subspace $\mathcal{U}\left(\alpha_{i}\right) \oplus \mathcal{U}\left(\alpha_{j}\right) \oplus \mathcal{U}\left(\alpha_{i}+\alpha_{j}\right)$ of $\widehat{\mathcal{U}}$, and let $\left\{c_{i}, c_{j}, c_{i j}, c_{j i}\right\}$ be the corresponding coordinate functions. Computing these coordinates on both sides of (3.1), we obtain:

$$
c_{i}=t_{1}+t_{3}=p_{2}, c_{j}=t_{2}=p_{1}+p_{3}, c_{i j}=t_{1} t_{2}=p_{2} p_{3},
$$

which implies (3.3).

(b) Consider the subspace $\mathcal{U}\left(\alpha_{i}\right) \oplus \mathcal{U}\left(\alpha_{j}\right) \oplus \mathcal{U}\left(\alpha_{i}+\alpha_{j}\right) \oplus \mathcal{U}\left(2 \alpha_{i}+\alpha_{j}\right)$ of $\widehat{\mathcal{U}}$. Since the Serre relations between $e_{i}$ and $e_{j}$ live in degrees $3 \alpha_{i}+\alpha_{j}$ and $\alpha_{i}+2 \alpha_{j}$, 
they do not affect this subspace, so as its basis we can take all non-commutative monomials in $e_{i}$ and $e_{j}$ of given degrees. Let $c_{i}, c_{j}, c_{i j}$, and $c_{i i j}$ be the coordinate functions corresponding to the basis vectors $e_{i}, e_{j}, e_{i} e_{j}$, and $e_{i}^{(2)} e_{j}$ respectively. Computing these coordinates on both sides of (3.1), we obtain:

$$
\begin{gathered}
c_{i}=t_{1}+t_{3}=p_{2}+p_{4}, c_{j}=t_{2}+t_{4}=p_{1}+p_{3}, \\
c_{i j}=t_{1} t_{2}+\left(t_{1}+t_{3}\right) t_{4}=p_{2} p_{3}, c_{i i j}=t_{1}^{2} t_{2}+\left(t_{1}+t_{3}\right)^{2} t_{4}=p_{2}^{2} p_{3},
\end{gathered}
$$

which easily implies (3.4).

(c) The same argument as in (b) provides five relations between the $p_{k}$ and the $t_{k}$ given by

$$
\begin{gathered}
c_{i}=t_{1}+t_{3}+t_{5}=p_{2}+p_{4}+p_{6}, c_{j}=t_{2}+t_{4}+t_{6}=p_{1}+p_{3}+p_{5}, \\
c_{i j}=t_{1} t_{2}+\left(t_{1}+t_{3}\right) t_{4}+\left(t_{1}+t_{3}+t_{5}\right) t_{6}=p_{2} p_{3}+\left(p_{2}+p_{4}\right) p_{5}, \\
c_{i i j}=t_{1}^{2} t_{2}+\left(t_{1}+t_{3}\right)^{2} t_{4}+\left(t_{1}+t_{3}+t_{5}\right)^{2} t_{6}=p_{2}^{2} p_{3}+\left(p_{2}+p_{4}\right)^{2} p_{5}, \\
c_{i i i j}=t_{1}^{3} t_{2}+\left(t_{1}+t_{3}\right)^{3} t_{4}+\left(t_{1}+t_{3}+t_{5}\right)^{3} t_{6}=p_{2}^{3} p_{3}+\left(p_{2}+p_{4}\right)^{3} p_{5} .
\end{gathered}
$$

To obtain a sixth relation, consider the component $\mathcal{U}\left(3 \alpha_{i}+2 \alpha_{j}\right)$. To analyze this subspace we need to take into account the Serre relation $\left(\operatorname{ad} e_{j}\right)^{2} e_{i}=0$, which can also be written in the form

$$
e_{j} e_{i} e_{j}=e_{j}^{(2)} e_{i}+e_{i} e_{j}^{(2)} .
$$

It is easy to see that $\operatorname{dim} \mathcal{U}\left(3 \alpha_{i}+2 \alpha_{j}\right)=7$. In fact, there are 10 non-commutative monomials of multi-degree $(3,2)$ in $e_{i}$ and $e_{j}$ but, in view of the above Serre relation, each of the 3 monomials $e_{i}^{(2)} e_{j} e_{i} e_{j}, e_{i} e_{j} e_{i} e_{j} e_{i}$, and $e_{j} e_{i} e_{j} e_{i}^{(2)}$ that contain a factor $e_{j} e_{i} e_{j}$ is a linear combination of the remaining 7 monomials. To be more precise, $e_{i}^{(2)} e_{j} e_{i} e_{j}$ is a linear combination of $e_{i}^{(2)} e_{j}^{(2)} e_{i}$ and $e_{i}^{(3)} e_{j}^{(2)}$, while $e_{i} e_{j} e_{i} e_{j} e_{i}$ is a linear combination of $e_{i} e_{j}^{(2)} e_{i}^{(2)}$ and $e_{i}^{(2)} e_{j}^{(2)} e_{i}$, and $e_{j} e_{i} e_{j} e_{i}^{(2)}$ is a linear combination of $e_{j}^{(2)} e_{i}^{(3)}$ and $e_{i} e_{j}^{(2)} e_{i}^{(2)}$. Note that none of these linear combinations involve the monomial $e_{i} e_{j} e_{i}^{(2)} e_{j}$. Hence the corresponding coordinate function $c_{i j i i j}$ can be computed as in the free algebra generated by $e_{i}$ and $e_{j}$. Computing this coordinate on both sides of (3.1), we obtain the desired sixth relation between the $p_{k}$ and the $t_{k}$ :

$$
c_{i j i i j}=t_{1} t_{2} t_{3}^{2} t_{4}+t_{1} t_{2}\left(t_{3}+t_{5}\right)^{2} t_{6}+\left(t_{1}+t_{3}\right) t_{4} t_{5}^{2} t_{6}=p_{2} p_{3} p_{4}^{2} p_{5} .
$$

The relations (3.11) through (3.15) provide a system of 6 polynomial equations with 6 unknowns $p_{1}, \ldots, p_{6}$. This system was solved using Maple, and the solution is given by (3.6). In a more old fashioned way, the system can be solved as follows. 
First, comparing (3.7) and (3.15), we see that

$$
\pi_{1}=c_{i j i i j}=p_{2} p_{3} p_{4}^{2} p_{5} .
$$

Next, consider the expression $\delta=c_{i j} c_{i i i j}-c_{i i j}^{2}$. An easy calculation using (3.12), (3.13) and (3.14) shows that

$$
\begin{gathered}
\delta=t_{1} t_{2} t_{3}^{2} t_{4}\left(t_{1}+t_{3}\right)+t_{1} t_{2}\left(t_{3}+t_{5}\right)^{2} t_{6}\left(t_{1}+t_{3}+t_{5}\right)+\left(t_{1}+t_{3}\right) t_{4} t_{5}^{2} t_{6}\left(t_{1}+t_{3}+t_{5}\right) \\
=p_{2} p_{3} p_{4}^{2} p_{5}\left(p_{2}+p_{4}\right) .
\end{gathered}
$$

Now a direct check shows that

$$
\begin{gathered}
\pi_{2}=c_{i j} \delta-c_{i i j} \pi_{1}=p_{2}^{2} p_{3}^{2} p_{4}^{3} p_{5}, \\
\pi_{3}=c_{i i j} \delta-c_{i i i j} \pi_{1}=p_{2}^{3} p_{3}^{2} p_{4}^{3} p_{5}, \\
\pi_{4}=\pi_{2} \delta-\pi_{1} \pi_{3}=p_{2}^{3} p_{3}^{3} p_{4}^{6} p_{5}^{2}, \\
t_{1} t_{2} t_{3}^{2} t_{4} t_{5}=c_{i} \pi_{1}-\delta=p_{2} p_{3} p_{4}^{2} p_{5} p_{6}, \\
t_{2} t_{3}^{3} t_{4}^{2} t_{5}^{3} t_{6}=c_{j} \pi_{3}-c_{i j} \pi_{2}+\pi_{1}^{2}=p_{1} p_{2}^{3} p_{3}^{2} p_{4}^{3} p_{5} .
\end{gathered}
$$

The right hand sides of (3.16) and (3.18) - (3.22) are monomials in $p_{1}, \ldots, p_{6}$, and an easy check shows that they are related to $p_{1}, \ldots, p_{6}$ by an invertible monomial transformation. Computing the inverse of this transformation yields (3.6), thus completing the proof of Theorem 3.1.

Remark 3.2. It is easy to see that in each of the cases in Theorem 3.1, the formulas expressing the $t_{k}$ through the $p_{k}$ (i.e., the inverse transformation $R_{j i j \ldots . . .}^{i j i \ldots}$ ) can be found by the substitution $p_{k} \mapsto t_{d+1-k}, t_{k} \mapsto p_{d+1-k}$ in (3.3), (3.4), or (3.6).

Remark 3.3. Note that, in each of the cases, our formulas for the transition map $R_{i j i \ldots}^{j i j \ldots}$ imply two "monomial" relations: for $d=2$ they are given by $(3.2)$, and in the other cases they are as follows:

$$
\begin{gathered}
t_{1} t_{2}=p_{2} p_{3}, t_{2} t_{3}=p_{1} p_{2} \quad(d=3) ; \\
t_{1} t_{2} t_{3}=p_{2} p_{3} p_{4}, t_{2} t_{3}^{2} t_{4}=p_{1} p_{2}^{2} p_{3} \quad(d=4) ; \\
t_{1} t_{2} t_{3}^{2} t_{4} t_{5}=p_{2} p_{3} p_{4}^{2} p_{5} p_{6}, t_{2} t_{3}^{3} t_{4}^{2} t_{5}^{3} t_{6}=p_{1} p_{2}^{3} p_{3}^{2} p_{4}^{3} p_{5} \quad(d=6) .
\end{gathered}
$$

Remark 3.4. Since all the expressions in (3.2) - (3.10) are subtraction-free, Theorem 3.1 implies Theorem 1.6 from the introduction. 


\section{Chamber Ansatz}

The main result of this section is a generalization of Theorems 2.7.1 and 5.3.2 from [2]. The terminology below is suggested by the $A_{r}$ case treated in [2]. In what follows we fix an element $w \in W$ of length $l(w)=m$. As explained in the introduction, Theorem 1.6 allows us to associate to $w$ the Lusztig variety $\mathcal{L}^{w}=$ $\mathcal{L}^{w}(K)$ defined over an arbitrary semifield $K$. As in [2], Section 2.1, by a semifield we mean a set $K$ endowed with two operations, addition and multiplication, which have the following properties:

addition in $K$ is commutative and associative;

multiplication makes $K$ an abelian group;

distributivity: $(a+b) c=a c+b c$ for $a, b, c \in K$.

Recall from the introduction that an element $\mathbf{t}$ of $\mathcal{L}^{w}$ is a tuple $\mathbf{t}=\left(t^{\mathbf{i}}\right) \mathbf{i} \in R(w)$, where each $t^{\mathbf{i}}=\left(t_{1}^{\mathbf{i}}, \ldots, t_{m}^{\mathbf{i}}\right)$ is a "vector" in $K^{m}$, and these vectors satisfy the relations $t^{\mathbf{i}^{\prime}}=R_{\mathbf{i}}^{\mathbf{i}^{\prime}}\left(t^{\mathbf{i}}\right)$ for all $\mathbf{i}, \mathbf{i}^{\prime} \in R(w)$. The following proposition generalizes [2], Theorem 2.2.6 and is proved in the same way.

Proposition 4.1. For any $\mathbf{i} \in R(w)$, the projection $\mathbf{t} \mapsto t^{\mathbf{i}}$ is a bijection between the Lusztig variety $\mathcal{L}^{w}(K)$ and $K^{m}$.

We will now present an alternative description of $\mathcal{L}^{w}$ that generalizes the one in [2], Sections 2.7 and 5.3. We will use the terminology and notation from Section 2. Let us introduce the variables $M_{\gamma}\left(\gamma \in E^{w}\right)$ labeled by $w$-chamber weights and taking values in $K$. We will express the components $t_{k}^{\mathbf{i}}$ of an element of the Lusztig variety by means of the substitution

$$
t_{k}^{\mathrm{i}}=\frac{1}{M_{w_{k}^{\mathrm{i}} \omega_{i_{k}}} M_{w_{k+1}^{\mathrm{i}} \omega_{i_{k}}}} \prod_{j \neq i_{k}} M_{w_{k}^{\mathrm{i}} \omega_{j}}^{-a_{j, i_{k}}}
$$

In the case of type $A_{r}$, this substitution reduces to the Chamber Ansatz in [2], (2.5.3). We will call (4.4) the generalized Chamber Ansatz. The following proposition generalizes [2], Proposition 2.5.1.

Proposition 4.2. The point $\mathbf{t}=\left(t_{k}^{\mathbf{i}}\right)$ whose components are defined by the generalized Chamber Ansatz (4.4) belongs to the Lusztig variety $\mathcal{L}^{w}(K)$ if and only if the $M_{\gamma}$ satisfy the following relations (4.5)-(4.11).

Case $d=3$. Suppose $a_{i j}=a_{j i}=-1$, and $w^{\prime} \in W$ is such that $l\left(w w^{\prime} s_{i} s_{j} s_{i}\right)=$ $l(w)-l\left(w^{\prime} s_{i} s_{j} s_{i}\right)=l(w)-l\left(w^{\prime}\right)-3$. Then

$$
M_{w^{\prime} s_{i} \omega_{i}} M_{w^{\prime} s_{j} \omega_{j}}=M_{w^{\prime} \omega_{i}} M_{w^{\prime} s_{i} s_{j} \omega_{j}}+M_{w^{\prime} s_{j} s_{i} \omega_{i}} M_{w^{\prime} \omega_{j}} .
$$


Case $d=4$. Suppose $a_{i j}=-2, a_{j i}=-1$, and $w^{\prime} \in W$ is such that $l\left(w w^{\prime} s_{i} s_{j} s_{i} s_{j}\right)=l(w)-l\left(w^{\prime} s_{i} s_{j} s_{i} s_{j}\right)=l(w)-l\left(w^{\prime}\right)-4$. Then

$$
\begin{gathered}
M_{w^{\prime} s_{i} \omega_{i}} M_{w^{\prime} s_{j} s_{i} \omega_{i}} M_{w^{\prime} s_{j} \omega_{j}}=M_{w^{\prime} s_{j} s_{i} \omega_{i}}^{2} M_{w^{\prime} \omega_{j}} \\
+\left(M_{w^{\prime} \omega_{i}} M_{w^{\prime} s_{j} s_{i} s_{j} \omega_{j}}+M_{w^{\prime} s_{i} s_{j} s_{i} \omega_{i}} M_{w^{\prime} s_{j} \omega_{j}}\right) M_{w^{\prime} \omega_{i}} \\
M_{w^{\prime} s_{i} s_{j} \omega_{j}} M_{w^{\prime} s_{j} s_{i} \omega_{i}}^{2} M_{w^{\prime} s_{j} \omega_{j}}=M_{w^{\prime} s_{j} s_{i} s_{j} \omega_{j}} M_{w^{\prime} s_{j} s_{i} \omega_{i}}^{2} M_{w^{\prime} \omega_{j}} \\
+\left(M_{w^{\prime} \omega_{i}} M_{w^{\prime} s_{j} s_{i} s_{j} \omega_{j}}+M_{w^{\prime} s_{i} s_{j} s_{i} \omega_{i}} M_{w^{\prime} s_{j} \omega_{j}}\right)^{2} ;
\end{gathered}
$$

Case $d=6$. Suppose $a_{i j}=-3, a_{j i}=-1$, and $w^{\prime} \in W$ is such that

$$
l\left(w w^{\prime} s_{i} s_{j} s_{i} s_{j} s_{i} s_{j}\right)=l(w)-l\left(w^{\prime} s_{i} s_{j} s_{i} s_{j} s_{i} s_{j}\right)=l(w)-l\left(w^{\prime}\right)-6 .
$$

Then

$$
\begin{aligned}
& M_{w^{\prime} s_{i} \omega_{i}} M_{w^{\prime} s_{j} s_{i} \omega_{i}}^{2} M_{w^{\prime} s_{j} s_{i} s_{j} s_{i} \omega_{i}} M_{w^{\prime} s_{j} \omega_{j}} M_{w^{\prime} s_{j} s_{i} s_{j} \omega_{j}} \\
& =M_{w^{\prime} s_{j} s_{i} \omega_{i}}^{3} M_{w^{\prime} s_{j} s_{i} s_{j} s_{i} \omega_{i}} M_{w^{\prime} \omega_{j}} M_{w^{\prime} s_{j}} s_{i} s_{j} \omega_{j} \\
& +\left(M_{w^{\prime} \omega_{i}} M_{w^{\prime} s_{j} s_{i} s_{j} \omega_{j}}+M_{w^{\prime} s_{j} s_{i} s_{j} s_{i} \omega_{i}} M_{w^{\prime} s_{j} \omega_{j}}\right)^{2} M_{w^{\prime} \omega_{i}} M_{w^{\prime} s_{j} s_{i} s_{j} s_{i} \omega_{i}} \\
& +\left(M_{w^{\prime} s_{j} s_{i} \omega_{i}} M_{w^{\prime} s_{j} s_{i} s_{j} s_{i} s_{j} \omega_{j}}+M_{w^{\prime} s_{i} s_{j} s_{i} s_{j} s_{i} \omega_{i}} M_{w^{\prime} s_{j} s_{i} s_{j} \omega_{j}}\right) M_{w^{\prime} \omega_{i}} M_{w^{\prime} s_{j} s_{i} \omega_{i}}^{2} M_{w^{\prime} s_{j} \omega_{j}} \text {; } \\
& M_{w^{\prime} s_{i} s_{j} s_{i} \omega_{i}} M_{w^{\prime} s_{j} s_{i} \omega_{i}}^{3} M_{w^{\prime} s_{j} s_{i} s_{j} s_{i} \omega_{i}}^{2} M_{w^{\prime} s_{j} \omega_{j}} M_{w^{\prime} s_{j} s_{i} s_{j} \omega_{j}}^{2} \\
& =M_{w^{\prime} s_{j} s_{i} \omega_{i}}^{3} M_{w^{\prime} s_{j} s_{i} s_{j} s_{i} \omega_{i}}^{3} M_{w^{\prime} \omega_{j}} M_{w^{\prime} s_{j} s_{i} s_{j} \omega_{j}}^{2} \\
& +\left(M_{w^{\prime} \omega_{i}} M_{w^{\prime} s_{j} s_{i} s_{j} \omega_{j}}+M_{w^{\prime} s_{j} s_{i} s_{j} s_{i} \omega_{i}} M_{w^{\prime} s_{j} \omega_{j}}\right)^{3} M_{w^{\prime} s_{j} s_{i} s_{j} s_{i} \omega_{i}}^{3} \\
& +\left(M_{w^{\prime} s_{j} s_{i} \omega_{i}} M_{w^{\prime} s_{j} s_{i} s_{j} s_{i} s_{j} \omega_{j}}+M_{w^{\prime} s_{i} s_{j} s_{i} s_{j} s_{i} \omega_{i}} M_{w^{\prime} s_{j} s_{i} s_{j} \omega_{j}}\right)^{2} M_{w^{\prime} s_{j} s_{i} \omega_{i}}^{4} M_{w^{\prime} s_{j} \omega_{j}} \\
& +\left(3 M_{w^{\prime} \omega_{i}} M_{w^{\prime} s_{j} s_{i} \omega_{i}} M_{w^{\prime} s_{j} s_{i} s_{j} \omega_{j}} M_{w^{\prime} s_{j} s_{i} s_{j} s_{i} s_{j} \omega_{j}}+2 M_{w^{\prime} \omega_{i}} M_{w^{\prime} s_{i} s_{j} s_{i} s_{j} s_{i} \omega_{i}} M_{w^{\prime} s_{j} s_{i} s_{j} \omega_{j}}^{2}\right. \\
& +2 M_{w^{\prime} s_{j} s_{i} s_{j} s_{i} \omega_{i}} M_{w^{\prime} s_{i} s_{j} s_{i} s_{j} s_{i} \omega_{i}} M_{w^{\prime} s_{j} \omega_{j}} M_{w^{\prime} s_{j} s_{i} s_{j} \omega_{j}} \\
& \left.+2 M_{w^{\prime} s_{j} s_{i} \omega_{i}} M_{w^{\prime} s_{j} s_{i} s_{j} s_{i} \omega_{i}} M_{w^{\prime} s_{j} \omega_{j}} M_{w^{\prime} s_{j} s_{i} s_{j} s_{i} s_{j} \omega_{j}}\right) \cdot M_{w^{\prime} s_{j} s_{i} \omega_{i}}^{2} M_{w^{\prime} s_{j} s_{i} s_{j} s_{i} \omega_{i}}^{2} M_{w^{\prime} s_{j} \omega_{j}} \text {; } \\
& M_{w^{\prime} s_{i} s_{j} s_{i} s_{j} \omega_{j}} M_{w^{\prime} s_{j} s_{i} \omega_{i}}^{3} M_{w^{\prime} s_{j} s_{i} s_{j} s_{i} \omega_{i}}^{3} M_{w^{\prime} s_{j} \omega_{j}} M_{w^{\prime} s_{j} s_{i} s_{j} \omega_{j}}^{2} \\
& =M_{w^{\prime} s_{j} s_{i} \omega_{i}}^{3} M_{w^{\prime} s_{j} s_{i} s_{j} s_{i} \omega_{i}}^{3} M_{w^{\prime} \omega_{j}} M_{w^{\prime} s_{j} s_{i} s_{j} \omega_{j}}^{2} M_{w^{\prime} s_{j} s_{i} s_{j} s_{i} s_{j} \omega_{j}} \\
& +\left(M_{w^{\prime} \omega_{i}} M_{w^{\prime} s_{j} s_{i} s_{j} \omega_{j}}+M_{w^{\prime} s_{j} s_{i} s_{j} s_{i} \omega_{i}} M_{w^{\prime} s_{j} \omega_{j}}\right)^{3} M_{w^{\prime} s_{j} s_{i} s_{j} s_{i} \omega_{i}}^{3} M_{w^{\prime} s_{j} s_{i} s_{j} s_{i} s_{j} \omega_{j}} \\
& +\left(M_{w^{\prime} s_{j} s_{i} \omega_{i}} M_{w^{\prime} s_{j} s_{i} s_{j} s_{i} s_{j} \omega_{j}}+M_{w^{\prime} s_{i} s_{j} s_{i} s_{j} s_{i} \omega_{i}} M_{w^{\prime} s_{j} s_{i} s_{j} \omega_{j}}\right)^{3} M_{w^{\prime} s_{j} s_{i} \omega_{i}}^{3} M_{w^{\prime} s_{j} \omega_{j}} \\
& +\left(3 M_{w^{\prime} \omega_{i}} M_{w^{\prime} s_{j} s_{i} \omega_{i}} M_{w^{\prime} s_{j} s_{i} s_{j} \omega_{j}} M_{w^{\prime} s_{j} s_{i} s_{j} s_{i} s_{j} \omega_{j}}+3 M_{w^{\prime} \omega_{i}} M_{w^{\prime} s_{i} s_{j} s_{i} s_{j} s_{i} \omega_{i}} M_{w^{\prime} s_{j} s_{i} s_{j} \omega_{j}}^{2}\right. \\
& +3 M_{w^{\prime} s_{j} s_{i} s_{j} s_{i} \omega_{i}} M_{w^{\prime} s_{i} s_{j} s_{i} s_{j} s_{i} \omega_{i}} M_{w^{\prime} s_{j} \omega_{j}} M_{w^{\prime} s_{j}} s_{i} s_{j} \omega_{j} \\
& \left.+2 M_{w^{\prime} s_{j} s_{i} \omega_{i}} M_{w^{\prime} s_{j} s_{i} s_{j} s_{i} \omega_{i}} M_{w^{\prime} s_{j} \omega_{j}} M_{w^{\prime} s_{j} s_{i} s_{j} s_{i} s_{j} \omega_{j}}\right) \\
& \cdot M_{w^{\prime} s_{j} s_{i} \omega_{i}}^{2} M_{w^{\prime} s_{j} s_{i} s_{j} s_{i} \omega_{i}}^{2} M_{w^{\prime} s_{j} \omega_{j}} M_{w^{\prime} s_{j} s_{i} s_{j} s_{i} s_{j} \omega_{j}} \text {; }
\end{aligned}
$$




$$
\begin{aligned}
& M_{w^{\prime} s_{i} s_{j} \omega_{j}} M_{w^{\prime} s_{j} s_{i} \omega_{i}}^{6} M_{w^{\prime} s_{j} s_{i} s_{j} s_{i} \omega_{i}}^{3} M_{w^{\prime} s_{j} \omega_{j}}^{2} M_{w^{\prime} s_{j} s_{i} s_{j} \omega_{j}}^{3} \\
& =\left\{M_{w^{\prime} s_{j} s_{i} \omega_{i}}^{3} M_{w^{\prime} s_{j} s_{i} s_{j} s_{i} \omega_{i}} M_{w^{\prime} \omega_{j}} M_{w^{\prime} s_{j} s_{i} s_{j} \omega_{j}}^{2}\right. \\
& +2\left(M_{w^{\prime} \omega_{i}} M_{w^{\prime} s_{j} s_{i} s_{j} \omega_{j}}+M_{w^{\prime} s_{j} s_{i} s_{j} s_{i} \omega_{i}} M_{w^{\prime} s_{j} \omega_{j}}\right)^{3} M_{w^{\prime} s_{j} s_{i} s_{j} s_{i} \omega_{i}} \\
& +\left(3 M_{w^{\prime} \omega_{i}} M_{w^{\prime} s_{j} s_{i} \omega_{i}} M_{w^{\prime} s_{j} s_{i} s_{j} \omega_{j}} M_{w^{\prime} s_{j} s_{i} s_{j} s_{i} s_{j} \omega_{j}}+3 M_{w^{\prime} \omega_{i}} M_{w^{\prime} s_{i} s_{j} s_{i} s_{j} s_{i} \omega_{i}} M_{w^{\prime} s_{j} s_{i} s_{j} \omega_{j}}^{2}\right. \\
& +3 M_{w^{\prime} s_{j} s_{i} s_{j} s_{i} \omega_{i}} M_{w^{\prime} s_{i} s_{j} s_{i} s_{j} s_{i} \omega_{i}} M_{w^{\prime} s_{j} \omega_{j}} M_{w^{\prime} s_{j} s_{i} s_{j} \omega_{j}} \\
& \left.\left.+2 M_{w^{\prime} s_{j} s_{i} \omega_{i}} M_{w^{\prime} s_{j} s_{i} s_{j} s_{i} \omega_{i}} M_{w^{\prime} s_{j} \omega_{j}} M_{w^{\prime} s_{j} s_{i} s_{j} s_{i} s_{j} \omega_{j}}\right) M_{w^{\prime} s_{j} s_{i} \omega_{i}}^{2} M_{w^{\prime} s_{j} \omega_{j}}\right\} \\
& +M_{w^{\prime} s_{j} s_{i} \omega_{i}}^{3} M_{w^{\prime} s_{j} s_{i} s_{j} s_{i} \omega_{i}}^{2} M_{\omega_{j}} M_{w^{\prime} s_{j} s_{i} s_{j} \omega_{j}}^{2} \\
& +\left\{\left(M_{w^{\prime} \omega_{i}} M_{w^{\prime} s_{j} s_{i} s_{j} \omega_{j}}+M_{w^{\prime} s_{j} s_{i} s_{j} s_{i} \omega_{i}} M_{w^{\prime} s_{j} \omega_{j}}\right)^{2} M_{w^{\prime} s_{j} s_{i} s_{j} s_{i} \omega_{i}}\right. \\
& \left.+\left(M_{w^{\prime} s_{j} s_{i} \omega_{i}} M_{w^{\prime} s_{j} s_{i} s_{j} s_{i} s_{j} \omega_{j}}+M_{w^{\prime} s_{i} s_{j} s_{i} s_{j} s_{i} \omega_{i}} M_{w^{\prime} s_{j} s_{i} s_{j} \omega_{j}}\right) M_{w^{\prime} s_{j} s_{i} \omega_{i}}^{2} M_{w^{\prime} s_{j} \omega_{j}}\right\}^{3} .
\end{aligned}
$$

The proof is straightforward: we only need to substitute the expressions given by (4.4) into the formulas (3.2), (3.3), (3.4), and (3.6), and clear the denominators. Note that the monomial relations referred to in Remark 3.3, will be fulfilled automatically, so each of the cases $d=2,3,4$, or 6 produces $d-2$ relations between the $M_{\gamma}$.

Generalizing [2], Section 2.7, we define $\tilde{\mathcal{M}}^{w}=\tilde{\mathcal{M}}^{w}(K)$ to be the set of all tuples $\mathbf{M}=\left(M_{\gamma}\right)_{\gamma \in E^{w}}$ of elements of the ground semifield $K$ satisfying the relations (4.5)-(4.11). According to Proposition 4.2, the generalized Chamber Ansatz (4.4) provides a well-defined map $\mathbf{M} \mapsto \mathbf{t}(\mathbf{M})$ from $\tilde{\mathcal{M}}^{w}(K)$ to $\mathcal{L}^{w}(K)$. Let $\mathcal{M}^{w}=$ $\mathcal{M}^{w}(K)$ be the subset of $\tilde{\mathcal{M}}^{w}$ formed by those tuples $\mathbf{M}=\left(M_{\gamma}\right)$ that, in addition to (4.5)-(4.11), satisfy the normalization condition

$$
M_{\omega_{i}}=1, \quad i=1, \ldots, r .
$$

The following theorem generalizes Theorem 2.7.1 in [2].

Theorem 4.3. The restriction to $\mathcal{M}^{w}$ of the map $\mathbf{M} \mapsto \mathbf{t}(\mathbf{M})$ is a bijection between $\mathcal{M}^{w}$ and the Lusztig variety $\mathcal{L}^{w}$. The inverse bijection $\mathbf{t}=\left(t_{k}^{\mathbf{i}}\right) \mapsto \mathbf{M}(\mathbf{t})=$ $\left(M_{\gamma}\right)$ from $\mathcal{L}^{w}$ to $\mathcal{M}^{w}$ is given as follows: if $\gamma=\gamma(k ; \mathbf{i})$ for some $\mathbf{i} \in R(w)$ and $k=1, \ldots, m$, then

$$
M_{\gamma}=M_{\gamma}(\mathbf{t})=\prod_{l \geq k}\left(t_{l}^{\mathbf{i}}\right)^{b(k, l ; \mathbf{i})},
$$

where $b(k, l ; \mathbf{i})=\left(\gamma(k ; \mathbf{i}),\left(\alpha_{l}^{\mathbf{i}}\right)^{\vee}\right)$.

Proof. First we will show that $M_{\gamma}$ is well defined via (4.13), i.e., that the right hand side of (4.13) does not depend on the choice of $\mathbf{i}$ and $k$ such that $\gamma=\gamma(k ; \mathbf{i})$. In view of Proposition 2.11, it is enough to show the following: if $\gamma=\gamma(k ; \mathbf{i})=\gamma\left(k^{\prime} ; \mathbf{i}^{\prime}\right)$, 
and $\mathbf{i}$ and $\mathbf{i}^{\prime}$ are related by a $d$-move, then

$$
\prod_{l \geq k}\left(t_{l}^{\mathbf{i}}\right)^{b(k, l ; \mathbf{i})}=\prod_{l \geq k^{\prime}}\left(t_{l}^{\mathbf{i}^{\prime}}\right)^{b\left(k^{\prime}, l ; \mathbf{i}^{\prime}\right)} .
$$

This is checked directly using the formulas for the transition maps in Theorem 3.1; to be more precise, we only need the "monomial" part of the transition maps given by $(3.2)$ and (3.23)-(3.25).

To complete the proof of Theorem 4.3, it remains to show that both compositions $\mathbf{M} \mapsto \mathbf{M}(\mathbf{t}(\mathbf{M}))$ and $\mathbf{t} \mapsto \mathbf{t}(\mathbf{M}(\mathbf{t}))$ are identity maps. In proving this, we will fix a reduced expression $\mathbf{i}=\left(i_{1}, \ldots, i_{m}\right) \in R(w)$ and will use a shorthand $M_{k}=M_{\gamma(k ; \mathbf{i})}, t_{k}=t_{k}^{\mathbf{i}}$, and $b(k, l)=b(k, l ; \mathbf{i})$. Using this notation, the correspondence $\mathbf{t} \mapsto \mathbf{M}(\mathbf{t})$ given by (4.13) can be written as

$$
M_{k}=\prod_{l \geq k} t_{l}^{b(k, l)},
$$

while the Chamber Ansatz correspondence $\mathbf{M} \mapsto \mathbf{t}(\mathbf{M})$ given by (4.4) can be written as

$$
t_{k}=\frac{1}{M_{k} M_{\nu\left(k ; i_{k}\right)}} \prod_{j \neq i_{k}} M_{\nu(k ; j)}^{-a_{j, i_{k}}},
$$

where $\nu(k ; j)=\min \left\{l: l>k, i_{l}=j\right\}$ (if $i_{l} \neq j$ for $l>k$ then we set $\nu(k ; j)=$ $m+1$ and $M_{m+1}=1$ ). The formulas (4.14) and (4.15) define two monomial transformations $K^{m} \rightarrow K^{m}$, and we only need to show that they are inverses of each other. Clearly, it is enough to show that one of their compositions is the identity map. So it suffices to prove the following identity for $k=1, \ldots, m$ :

$$
M_{k}=\prod_{l \geq k}\left(\frac{1}{M_{l} M_{\nu\left(l ; i_{l}\right)}} \prod_{j \neq i_{l}} M_{\nu(l ; j)}^{-a_{j, i_{l}}}\right)^{b(k, l)} .
$$

The exponent of $M_{k}$ on the right-hand side of (4.16) is equal to

$$
-b(k, k)=-\left(\gamma(k ; \mathbf{i}),\left(\alpha_{k}^{\mathbf{i}}\right)^{\vee}\right)=\left(\omega_{i_{k}}, \alpha_{i_{k}}^{\vee}\right)=1,
$$

as needed. It remains to show that for every $k^{\prime}>k$, the variable $M_{k^{\prime}}$ cancels out on the right-hand side of (4.16). Let $i_{k^{\prime}}=i$, and let $k^{\prime \prime}=\max \left\{l: k \leq l<k^{\prime}, i_{l}=i\right\}$ (if $i_{l} \neq i$ for $k \leq l<k^{\prime}$ then we set $k^{\prime \prime}=k-1$ and $b\left(k, k^{\prime \prime}\right)=0$ ). The exponent of $M_{k^{\prime}}$ on the right-hand side of (4.16) can be written as

$$
-\left(b\left(k, k^{\prime}\right)+b\left(k, k^{\prime \prime}\right)+\sum_{l: k^{\prime \prime}<l<k^{\prime}} a_{i, i_{l}} b(k, l)\right) .
$$

Remembering the definition of $b(k, l)$, we can rewrite $(4.17)$ as

$$
-\left(\gamma(k ; \mathbf{i}),\left(\alpha_{k^{\prime}}^{\mathbf{i}}\right)^{\vee}+\left(\alpha_{k^{\prime \prime}}^{\mathbf{i}}\right)^{\vee}+\sum_{l: k^{\prime \prime}<l<k^{\prime}} a_{i, i_{l}}\left(\alpha_{l}^{\mathbf{i}}\right)^{\vee}\right) .
$$


Acting on both arguments in the scalar product by $s_{i_{k}} \cdots s_{i_{m}}$, we can rewrite $(4.18)$ as $\left(\omega_{i_{k}}, \beta\right)$, where

$$
\beta=s_{i_{k}} \cdots s_{i_{k^{\prime}-1}} \alpha_{i}^{\vee}+s_{i_{k}} \cdots s_{i_{k^{\prime \prime}-1}} \alpha_{i}^{\vee}+\sum_{l: k^{\prime \prime}<l<k^{\prime}} s_{i_{k}} \cdots s_{i_{l-1}}\left(a_{i, i_{l}} \alpha_{i_{l}}^{\vee}\right)
$$

In view of (2.5), we have

$$
a_{i, i_{l}} \alpha_{i_{l}}^{\vee}=\alpha_{i}^{\vee}-s_{i_{l}} \alpha_{i}^{\vee}
$$

substituting this expression into (4.19), we see that the sum becomes telescoping, and $\beta=0$. This completes the proof of (4.16) and of Theorem 4.3.

The following corollary generalizes Corollary 2.7.4 in [2].

Corollary 4.4. For every $\mathbf{i} \in R(w)$, the components $M_{\gamma(k ; \mathbf{i})}(k=1, \ldots, m)$ form a system of independent coordinates on $\mathcal{M}^{w}(K)$, that is, they can be assigned arbitrary values in $K$, and the remaining components $M_{\gamma}$ of a point $\mathbf{M} \in \mathcal{M}^{w}(K)$ are expressed through them as subtraction-free rational expressions.

Proof. In the course of the proof of Theorem 4.3, we have shown that, for every $\mathbf{i} \in R(w)$, the components $t_{k}^{\mathbf{i}}$ of a point $\mathbf{t} \in \mathcal{L}^{w}(K)$ and the components $M_{\gamma(k ; \mathbf{i})}$ of the corresponding point $\mathbf{M} \in \mathcal{M}^{w}(K)$ are related to each other by an invertible monomial transformation (see (4.14) and (4.15)). To prove our corollary, it remains to observe that by Proposition 4.1 , the $t_{k}^{\mathbf{i}}$ for any given $\mathbf{i} \in R(w)$ form a system of independent coordinates on $\mathcal{L}^{w}(K)$, and the remaining components $t_{k}^{\mathbf{i}^{\prime}}$ are expressed through them via the transition maps $R_{\mathbf{i}}^{\mathbf{i}^{\prime}}$ which are given by subtraction-free rational expressions.

\section{Proofs of theorem 1.2 and proposition 1.3}

As in the introduction, we consider the open subset $N^{w}=N \cap B_{-} w B_{-}$of the Schubert cell $C_{w}$. The involutive antiautomorphisms $x \mapsto x^{T}$ and $x \mapsto x^{\iota}$ of $G$ (see (1.6), (1.7)) preserve $H$ and $\operatorname{Norm}_{G}(H)$, and so act on $W$. We will denote by $\bar{w}$ a representative of $w \in W$ in $\operatorname{Norm}_{G}(H)$; a direct check shows that

$$
\bar{w}^{T}=\bar{w}^{\iota}=\overline{w^{-1}} .
$$

For any $w \in W$, we define the subgroups $N(w)$ and $N^{\prime}(w)$ of $N$ by

$$
N(w)=N \cap \bar{w}^{-1} N_{-} \bar{w}, N^{\prime}(w)=N \cap \bar{w}^{-1} N \bar{w} .
$$

The following two facts are well-known: 
the map $(x, y) \mapsto x y$ induces a bijection $N(w) \times N^{\prime}(w) \rightarrow N$;

the map $(x, y) \mapsto x \bar{w} y^{T}$ induces a bijection $B_{-} \times N(w) \rightarrow B_{-} w B_{-}$.

Now we are ready to introduce the map $\eta_{w}$. We will do this in a slightly more general setting than in Theorem 1.2.

Proposition 5.1. For every $z \in N^{w} \cdot N^{\prime}(w)$, the intersection $N \cap B_{-} w z^{T}$ consists of a unique element $x=\eta_{w}(z)$, so the correspondence $z \mapsto \eta_{w}(z)$ is a map from $N^{w} \cdot N^{\prime}(w)$ to $N^{w}$. For $y, z \in N^{w} \cdot N^{\prime}(w)$, the condition $\eta_{w}(y)=\eta_{w}(z)$ is equivalent to $y^{-1} z \in N^{\prime}(w)$.

Proof. The uniqueness of $x$ and the fact that $x \in N^{w}$ are obvious. To prove the existence of $x$, it is enough to show that $\bar{w} z^{T} \in B_{-} N$ for all $z \in N^{w} \cdot N^{\prime}(w)$. Since $\left(B_{-} N\right)^{T}=B_{-} N$ and $\left(\bar{w} z^{T}\right)^{T}=z \overline{w^{-1}}$ (see (5.1)), we only need to show that $N^{w} \cdot N^{\prime}(w) \bar{w}^{-1} \subset B_{-} N$. Using (5.3) and (5.4), we obtain

$$
N^{w} \cdot N^{\prime}(w) \bar{w}^{-1} \subset B_{-} \bar{w} N(w)^{T} N^{\prime}(w) \bar{w}^{-1} \subset B_{-} \bar{w} \cdot\left(\bar{w}^{-1} N \bar{w}\right) \bar{w}^{-1}=B_{-} N,
$$

as desired. Finally, the condition $\eta_{w}(y)=\eta_{w}(z)$ can be rewritten as $\bar{w} y^{T}\left(\bar{w} z^{T}\right)^{-1} \in$ $B_{-}$, which is easily seen to be equivalent to $y^{-1} z \in N^{\prime}(w)$. This completes the proof of Proposition 5.1.

We now turn to the proof of Proposition 1.3. The fact that the map $x \mapsto x^{\iota}$ restricts to a bijection $N^{w} \rightarrow N^{w^{-1}}$ follows at once from the definition (1.7) and from (5.1). Furthermore, (1.7) readily implies that, for any $\mathbf{i}=\left(i_{1}, \ldots, i_{m}\right) \in$ $R(w)$, we have

$$
x_{\mathbf{i}}\left(t_{1}, \ldots, t_{m}\right)^{\iota}=x_{\mathbf{i}^{*}}\left(t_{m}, \ldots, t_{1}\right),
$$

where $\mathbf{i}^{*}=\left(i_{m}, \ldots, i_{1}\right) \in R\left(w^{-1}\right)$. In view of Proposition 1.1, this shows that $x \mapsto x^{\iota}$ restricts to a bijection $N_{>0}^{w} \rightarrow N_{>0}^{w^{-1}}$, thus completing the proof of Proposition 1.3 (a).

Part (b) of Proposition 1.3 can be reformulated as follows.

Proposition 5.2. If $z \in N^{w}$ and $x=\eta_{w}(z)$ then $z^{\iota}=\eta_{w^{-1}}\left(x^{\iota}\right)$; therefore, $\eta_{w}: N^{w} \rightarrow N^{w}$ is a bijection.

Proof. According to (5.4), every $z \in N^{w}$ can be uniquely expressed as

$$
z=x^{T} \bar{w} y^{T} \quad(x \in N, y \in N(w)),
$$

where the choice of a representative $\bar{w}$ depends on $z$. We claim that the component $x$ in (5.6) is equal to $\eta_{w}(z)$. Indeed, (5.6) can be rewritten as $\bar{w} z^{T}=\bar{w} y \bar{w}^{T} x$; using (5.1), we see that $\bar{w} y \bar{w}^{T} \in B_{-}$, hence $x=\eta_{w}(z)$. 
Using (5.6), one can express $x$ as follows:

$$
x=u^{T}\left(\bar{w}^{T}\right)^{-1} z^{T}
$$

where $u=\bar{w}\left(y^{T}\right)^{-1} \bar{w}^{-1}$. Applying the anti-automorphism $x \mapsto x^{\iota}$ on both sides of (5.7), we obtain

$$
x^{\iota}=\left(z^{\iota}\right)^{T} \overline{w^{-1}}\left(u^{\iota}\right)^{T}
$$

(we used (5.1) and the obvious fact that $x \mapsto x^{\iota}$ and $x \mapsto x^{T}$ commute with each other). The condition that $y \in N(w)$ implies easily that $u \in N\left(w^{-1}\right)$, hence $u^{\iota} \in N\left(w^{-1}\right)$. It follows that (5.8) is an expression of the same kind as (5.6), with $z, x$, and $w$ replaced by $x^{\iota}, z^{\iota}$, and $w^{-1}$, respectively. Proposition 5.2 (and hence Proposition 1.3) follows.

To complete the proof of Theorem 1.2, it remains to show that the map $\eta_{w}$ : $N^{w} \rightarrow N^{w}$ sends $N_{>0}^{w}$ to itself. In view of Proposition 1.1, this is a consequence of the following.

Proposition 5.3. Let $\mathbf{i}=\left(i_{1}, \ldots, i_{m}\right) \in R(w)$ and $t_{1}, \ldots, t_{m}>0$. The map $\eta_{w}$ sends $x_{\mathbf{i}}\left(t_{1}, \ldots, t_{m}\right)$ to $x_{\mathbf{i}}\left(p_{1}, \ldots, p_{m}\right)$, where each $p_{k}$ is a subtraction-free rational expression in $t_{1}, \ldots, t_{m}$.

Proof. Let $z=x_{\mathbf{i}}\left(t_{1}, \ldots, t_{m}\right)$ and $x=\eta_{w}(z)$. As shown above, $x$ and $z$ are related by (5.6). To prove the equality $z=x_{\mathbf{i}}\left(p_{1}, \ldots, p_{m}\right)$, we proceed by induction on $m=l(w)$. If $m=1$ then the decomposition (5.6) takes the form

$$
x_{i}(t)=x_{i}\left(t^{-1}\right)^{T} \overline{s_{i}} x_{i}\left(t^{-1}\right)^{T},
$$

which can be checked by the matrix calculation in $S L_{2}$ :

$$
\left(\begin{array}{ll}
1 & t \\
0 & 1
\end{array}\right)=\left(\begin{array}{cc}
1 & 0 \\
t^{-1} & 1
\end{array}\right)\left(\begin{array}{cc}
0 & t \\
-t^{-1} & 0
\end{array}\right)\left(\begin{array}{cc}
1 & 0 \\
t^{-1} & 1
\end{array}\right)
$$

Thus, $\eta_{s_{i}}\left(x_{i}(t)\right)=x_{i}\left(t^{-1}\right)$.

For the induction step, we need some notation. For $i=1, \ldots, r$ and $t>0$ we set

$$
y_{i}(t)=x_{i}(t)^{T}=\exp \left(t f_{i}\right), t^{h_{i}}=\exp \left(\ln (t) h_{i}\right) ;
$$

thus, $y_{i}(t) \in N_{-}$and $t^{h_{i}} \in H$. These elements together with the $x_{i}(t)$ satisfy the following commutation relations:

$$
\begin{gathered}
p^{h_{i}} x_{j}(t)=x_{j}\left(p^{a_{i j}} t\right) p^{h_{i}}, p^{h_{i}} y_{j}(t)=y_{j}\left(p^{-a_{i j}} t\right) p^{h_{i}} \\
x_{i}(t) y_{j}(p)=y_{j}(p) x_{i}(t) \quad(i \neq j)
\end{gathered}
$$




$$
x_{i}(t) y_{i}(p)=y_{i}\left(p(1+t p)^{-1}\right)(1+t p)^{h_{i}} x_{i}\left(t(1+t p)^{-1}\right)
$$

(the relations (5.11) and (5.12) follow directly from the relations (1.3) in $\mathbf{g}$, and (5.13) can be proved by another matrix calculation in $S L_{2}$ ).

Now suppose that $m \geq 2$, and let $w^{\prime}=s_{i_{1}} w, \mathbf{i}^{\prime}=\left(i_{2}, \ldots, i_{m}\right) \in R\left(w^{\prime}\right), z^{\prime}=$ $x_{\mathbf{i}^{\prime}}\left(t_{2}, \ldots, t_{m}\right)$ and $x^{\prime}=\eta_{w^{\prime}}\left(z^{\prime}\right)$. By the inductive assumption, the decomposition (5.6) for $z^{\prime}$ has the form

$$
z^{\prime}=y_{i_{m}}\left(p_{m}^{\prime}\right) \cdots y_{i_{2}}\left(p_{2}^{\prime}\right) \overline{w^{\prime}} y^{\prime T}
$$

where $y^{\prime} \in N\left(w^{\prime}\right)$, and each $p_{k}^{\prime}$ is a subtraction-free rational expression in $t_{2}, \ldots, t_{m}$. Using (5.14) and the commutation relations $(5.11)-(5.13)$, we can rewrite $z=$ $x_{i_{1}}\left(t_{1}\right) z^{\prime}$ as follows:

$$
z=y_{i_{m}}\left(p_{m}\right) \cdots y_{i_{2}}\left(p_{2}\right) x_{i_{1}}(t) \overline{w^{\prime}} y^{\prime T}
$$

(to arrive at (5.15), we use (5.12) and (5.13) to push $x_{i_{1}}\left(t_{1}\right)$ to the right through all the terms $y_{i_{k}}\left(p_{k}^{\prime}\right)$ in (5.14); all the terms of the kind $t^{h_{i}}$ that are generated during this process with the help of (5.13) are then also moved to the right by applying (5.11)). Since the relations $(5.11)-(5.13)$ only involve subtraction-free expressions, we conclude that $t$ and all $p_{k}$ in (5.15) are subtraction-free rational expressions in $t_{1}, \ldots, t_{m}$. Using (5.9), we can rewrite the "tail" in (5.15) as follows:

$$
x_{i_{1}}(t) \overline{w^{\prime}} y^{\prime T}=y_{i_{1}}\left(t^{-1}\right) \overline{s_{i_{1}}} x_{i_{1}}\left(t^{-1}\right)^{T} \overline{w^{\prime}} y^{\prime T}=y_{i_{1}}\left(t^{-1}\right) \bar{w}\left(y^{\prime} y^{\prime \prime}\right)^{T}
$$

where $y^{\prime \prime}={\overline{w^{\prime}}}^{-1} x_{i_{1}}\left(t^{-1}\right) \overline{w^{\prime}}$. Clearly, $y^{\prime \prime}$ is a non-trivial element of the root subgroup $N\left(\alpha_{1}^{\mathbf{i}}\right) \subset N$, where $\alpha_{1}^{\mathbf{i}}=\left(w^{\prime}\right)^{-1}\left(\alpha_{i_{1}}\right)$ (cf. (2.8)). By Corollary 2.2, $y^{\prime} y^{\prime \prime} \in$ $N(w)$. Combining (5.15) and (5.16), we conclude that $\eta_{w}(z)=x_{i_{1}}\left(t^{-1}\right) x_{i_{2}}\left(p_{2}\right)$ $\cdots x_{i_{m}}\left(p_{m}\right)$, completing the proof of Proposition 5.3 and of Theorem 1.2.

The above proof yields the following corollary which will be used in the next section. Note that, in view of (5.4), for every $x \in N^{w}$ there is a unique $y \in N(w)$ such that $x \in B_{-} w y^{T}$, i.e., such that $x=\eta_{w}(y)$. Note also that, in view of Proposition 2.1 , for any $\mathbf{i}=\left(i_{1}, \ldots, i_{m}\right) \in R(w)$, every element $y \in N(w)$ has a unique factorization of the form

$$
y=y^{(m)} \cdots y^{(1)} \quad\left(y^{(k)} \in N\left(\alpha_{k}^{\mathbf{i}}\right)\right) .
$$

Proposition 5.4. Let $y \in N(w)$ be such that $\eta_{w}(y)=x_{\mathbf{i}}\left(t_{1}, \ldots, t_{m}\right) \in N_{>0}^{w}$ for some $\mathbf{i}=\left(i_{1}, \ldots, i_{m}\right) \in R(w)$ and $t_{1}, \ldots, t_{m}>0$. If $y$ is factored according to (5.17) then, for each $k=1, \ldots, m$, the element $y^{(m)} \cdots y^{(k)}$ belongs to $N\left(\left(w_{k}^{\mathbf{i}}\right)^{-1}\right)$ (see (2.7)), and $\eta_{\left(w_{k}^{\mathbf{i}}\right)^{-1}}\left(y^{(m)} \cdots y^{(k)}\right)=x_{i_{k}}\left(t_{k}\right) \cdots x_{i_{m}}\left(t_{m}\right)$.

Proof. In the notation of Proposition 5.3 and its proof, the element $y$ is expressed as $y^{\prime} y^{\prime \prime}$, where $y^{\prime \prime} \in N\left(\alpha_{1}^{\mathbf{i}}\right)$, and $y^{\prime} \in N\left(w^{\prime}\right)$ is such that $\eta_{w^{\prime}}\left(y^{\prime}\right)=x_{i_{2}}\left(t_{2}\right) \cdots x_{i_{m}}\left(t_{m}\right)$. Our statement follows by induction on $m$. 


\section{Totally positive varieties}

In this section we specialize the results of Section 4 to the case when the ground semifiled $K$ is $\mathbf{R}_{>0}$. In view of Proposition 1.1 , the Lusztig variety $\mathcal{L}^{w}\left(\mathbf{R}_{>0}\right)$ is naturally identified with the variety $N_{>0}^{w}$ of totally positive elements in the Schubert cell $C_{w}=B_{-} \backslash B_{-} w B_{-}$. Theorem 4.3 gives a bijection between $N_{>0}^{w}$ and the variety $\mathcal{M}^{w}\left(\mathbf{R}_{>0}\right)$, so each component $M_{\gamma}$ of a point from $\mathcal{M}^{w}\left(\mathbf{R}_{>0}\right)$ becomes a function on $N_{>0}^{w}$. Now Theorem 1.4 from the introduction can be reformulated as follows.

Theorem 6.1. For every $w$-chamber weight $\gamma$ the function $M_{\gamma}$ on $N_{>0}^{w}$ is given by $M_{\gamma}(x)=\Delta^{\gamma}(z)$, where $z=\eta_{w}^{-1}(x) \in N_{>0}^{w}$.

Before proving Theorem 6.1, we need to establish some results about the functions $\Delta^{\gamma}($ see $(1.9))$. To put these functions in a proper context, we recall some well-known facts from the representation theory of $G$. Let $P_{+}$be the additive semigroup in the weight lattice $P$ generated by the fundamental weights $\omega_{1}, \ldots, \omega_{r}$. The elements of $P_{+}$are highest weights of irreducible rational representations of $G$; we denote by $V_{\lambda}$ the representation with highest weight $\lambda$. Let $\mathbf{C}[G]$ be the ring of regular functions on $G$, and let $\mathbf{C}\left[N_{-} \backslash G\right]$ denote the subring of $\mathbf{C}[G]$ consisting of the functions $f$ such that $f(x g)=f(g)$ for all $x \in N_{-}$and $g \in G$. Both $\mathbf{C}[G]$ and $\mathbf{C}\left[N_{-} \backslash G\right]$ are $G$-modules under the action of $G$ on itself by right translations; so the action of $G$ on $\mathbf{C}[G]$ and $\mathbf{C}\left[N_{-} \backslash G\right]$ is given by

$$
(x f)(g)=f(g x), \quad(x, g \in G) .
$$

Each $V_{\lambda}$ has a canonical realization as a submodule of $\mathbf{C}\left[N_{-} \backslash G\right]$ :

$$
V_{\lambda}=\left\{f \in \mathbf{C}\left[N_{-} \backslash G\right]: f(d g)=d^{\lambda} f(g)(d \in H, g \in G)\right\} .
$$

These submodules are all irreducible components of $\mathbf{C}\left[N_{-} \backslash G\right]$ :

$$
\mathbf{C}\left[N_{-} \backslash G\right]=\oplus_{\lambda \in P_{+}} V_{\lambda} .
$$

For any $\lambda \in P_{+}$, we define a function $\Delta^{\lambda} \in \mathbf{C}\left[N_{-} \backslash G\right]$ as the highest vector in $V_{\lambda}$ normalized by the condition $\Delta^{\lambda}(e)=1$. In other words, $\Delta_{\lambda}$ is a regular function on $G$ whose restriction to the open set $N_{-} H N$ is given by

$$
\Delta^{\lambda}(p d x)=d^{\lambda}\left(p \in N_{-}, d \in H, x \in N\right) .
$$

Now let $\gamma=u \lambda$ for some $u \in W$ and $\lambda \in P_{+}$. Then $\gamma$ is an extremal weight for $V_{\lambda}$, and the corresponding weight subspace $V_{\lambda}(\gamma)$ is one-dimensional. We denote by $\Delta^{\gamma}$ an element of $V_{\lambda}(\gamma)$ (cf. (1.9)) normalized in the following way. Choose a reduced expression $\left(j_{1}, \ldots, j_{l}\right) \in R\left(u^{-1}\right)$ and for $k=1, \ldots, l$ set

$$
b_{k}=\left(s_{j_{k-1}} \cdots s_{j_{1}} \lambda, \alpha_{j_{k}}^{\vee}\right)=\left(\lambda, s_{j_{1}} \cdots s_{j_{k-1}} \alpha_{j_{k}}^{\vee}\right)
$$


It is well known that the action on $V_{\lambda}$ of the element $e_{j_{1}}^{\left(b_{1}\right)} \cdots e_{j_{l}}^{\left(b_{l}\right)} \in U(\mathbf{g})$ gives an isomorphism $V_{\lambda}(\gamma) \rightarrow V_{\lambda}(\lambda)$; here $e^{(b)}$ stands for the divided power $e^{b} / b$ !. We normalize $\Delta^{\gamma}$ by the condition

$$
e_{j_{1}}^{\left(b_{1}\right)} \cdots e_{j_{l}}^{\left(b_{l}\right)}\left(\Delta^{\gamma}\right)=\Delta^{\lambda}
$$

It is known that this normalization does not depend on the choice of an element $u \in W$ such that $\gamma=u \lambda$ and on the choice of a reduced expression $\left(j_{1}, \ldots, j_{l}\right) \in$ $R\left(u^{-1}\right)$ (see, e.g., [9], Section 28.1). The function $\Delta^{\gamma}$ can also be written as

$$
\Delta^{\gamma}=\bar{u} \Delta^{\lambda}
$$

for some specific choice of a representative $\bar{u}$. The normalization condition (6.6) implies that the representative $\bar{u}$ in (6.7) satisfies

$$
\bar{u}^{T}=\bar{u}^{-1}
$$

(indeed, using (6.6) and the representation theory of $S L_{2}$, we can write $\bar{u}$ as $\overline{s_{j_{l}}} \ldots \overline{s_{j_{1}}}$, where each $\overline{s_{j_{k}}}$ satisfies ${\overline{s_{k}}}^{T}={\overline{s_{j_{k}}}}^{-1}$ ).

We now turn to the proof of Theorem 6.1. First we will give its equivalent reformulation. The following theorem generalizes [2], Theorem 5.4.2.

Theorem 6.2. For every $w$-chamber weight $\gamma$, the function $M_{\gamma}$ on $N_{>0}^{w}$ is given by $M_{\gamma}(x)=\Delta^{\gamma}(y)$, where $y \in N(w)$ is such that $x=\eta_{w}(y)$.

In view of Proposition 5.2, the fact that Theorems 6.1 and 6.2 are equivalent to each other, is a consequence of the following statement.

Lemma 6.3. If $\gamma$ is a $w$-chamber weight then $\Delta^{\gamma}(z)=\Delta^{\gamma}(z p)$ for all $z \in G$ and $p \in N^{\prime}(w)$.

Proof. Every $w$-chamber weight can be written as $\gamma=u \lambda$, where $\lambda$ is some fundamental weight, and $u \in W$ satisfies $\operatorname{Inv}\left(u^{-1}\right) \subset \operatorname{Inv}(w)$ (see Corollary 2.3 and Proposition 2.10). The inclusion $\operatorname{Inv}\left(u^{-1}\right) \subset \operatorname{Inv}(w)$ readily implies that $N^{\prime}(w) \subset N^{\prime}\left(u^{-1}\right)$, i.e., that $\bar{u}^{-1} p \bar{u} \in N$ for all $p \in N^{\prime}(w)$. Using (6.7) and (6.4), we obtain

$$
\Delta^{\gamma}(z p)=\Delta^{\lambda}(z p \bar{u})=\Delta^{\lambda}\left(z \bar{u} \cdot \bar{u}^{-1} p \bar{u}\right)=\Delta^{\lambda}(z \bar{u})=\Delta^{\gamma}(z),
$$

as desired.

Turning to the proof of Theorem 6.2, we will first establish its special case when $\gamma=w^{-1} \omega_{i}$ for some $i$. Thus, we will prove the equality

$$
M_{w^{-1} \omega_{i}}(x)=\Delta^{w^{-1} \omega_{i}}(y),
$$


where $x$ and $y$ are as in Theorem 6.2. Choosing $\mathbf{i}=\left(i_{1}, \ldots, i_{m}\right) \in R(w)$ and writing $x$ as $x_{\mathbf{i}}\left(t_{1}, \ldots, t_{m}\right)$, we see that the left-hand side of $(6.9)$ is the monomial in the $t_{k}$ given by (4.13). In our situation, this monomial takes the following form:

$$
M_{w^{-1} \omega_{i}}(x)=\left(\prod_{k=1}^{m} t_{k}^{b_{k}}\right)^{-1}
$$

where the exponents $b_{k}$ are given by

$$
b_{k}=-\left(\gamma,\left(\alpha_{k}^{\mathbf{i}}\right)^{\vee}\right)=-\left(s_{i_{m}} \cdots s_{i_{1}} \omega_{i}, s_{i_{m}} \cdots s_{i_{k+1}} \alpha_{i_{k}}^{\vee}\right)=\left(\omega_{i}, s_{i_{1}} \cdots s_{i_{k-1}} \alpha_{i_{k}}^{\vee}\right) .
$$

In view of (6.10), we see that (6.9) becomes a consequence of the following lemma.

Lemma 6.4. Suppose $\gamma=w^{-1} \lambda$ for some $w \in W$ and $\lambda \in P_{+}$.

(a) Let $x \in N^{w}$ and $y \in N(w)$ be such that $x=\eta_{w}(y)$. Then

$$
\Delta^{\gamma}(y) \Delta^{\gamma}(x)=1
$$

(b) Let $x=x_{\mathbf{i}}\left(t_{1}, \ldots, t_{m}\right)$ for some $\mathbf{i}=\left(i_{1}, \ldots, i_{m}\right) \in R(w)$ and $t_{1}, \ldots, t_{m} \in$ C. Then

$$
\Delta^{\gamma}(x)=\prod_{k=1}^{m} t_{k}^{b_{k}},
$$

where the exponents $b_{k}$ are given by

$$
b_{k}=\left(\lambda, s_{i_{1}} \cdots s_{i_{k-1}} \alpha_{i_{k}}^{\vee}\right) .
$$

Proof. (a) Note that (6.11) generalizes [2], (5.4.6); our argument will be essentially the same as in [2]. In view of (6.7) and (6.8), one can choose a representative $\bar{w}$ so that $\bar{w} \Delta^{\gamma}=\Delta^{\lambda}$ and $\bar{w}^{T}=\bar{w}^{-1}$. Thus, we have $\Delta^{\gamma}=\bar{w}^{T} \Delta^{\lambda}$. By the definition of the map $\eta_{w}$, we have $\bar{w} y^{T}=p d x$ for some $p \in N_{-}$and $d \in H$. Since $\Delta^{\gamma} \in V_{\lambda}$, we conclude from (5.19) that

$$
\Delta^{\gamma}\left(\bar{w} y^{T}\right)=d^{\lambda} \Delta^{\gamma}(x)
$$

The same argument applied to $\Delta^{\lambda}$ yields

$$
\Delta^{\lambda}\left(\bar{w} y^{T}\right)=d^{\lambda} \Delta^{\lambda}(x)=d^{\lambda} .
$$

Comparing (6.14) and (6.15), we see that

$$
\Delta^{\lambda}\left(\bar{w} y^{T}\right) \Delta^{\gamma}(x)=\Delta^{\gamma}\left(\bar{w} y^{T}\right) .
$$


To deduce (6.11) from (6.16), it remains to notice that

$$
\Delta^{\lambda}\left(\bar{w} y^{T}\right)=\Delta^{\lambda}\left(y \bar{w}^{T}\right)=\left(\bar{w}^{T} \Delta^{\lambda}\right)(y)=\Delta^{\gamma}(y)
$$

(since, in view of (6.4), $\Delta^{\lambda}\left(z^{T}\right)=\Delta^{\lambda}(z)$ for all $\left.z \in G\right)$, and

$$
\Delta^{\gamma}\left(\bar{w} y^{T}\right)=\left(\bar{w} \Delta^{\gamma}\right)\left(\bar{w} y^{T} \bar{w}^{-1}\right)=\Delta^{\lambda}\left(\bar{w} y^{T} \bar{w}^{-1}\right)=1
$$

(since $\bar{w} y^{T} \bar{w}^{-1} \in N_{-}$whenever $y \in N(w)$ ).

(b) We will use the following general formula valid for any $f \in \mathbf{C}[G]$ and for any sequence of indices $\mathbf{i}=\left(i_{1}, \ldots, i_{m}\right)$ (not necessarily a reduced expression):

$$
f\left(x_{\mathbf{i}}\left(t_{1}, \ldots, t_{m}\right)\right)=\sum_{a_{1}, \ldots, a_{m} \geq 0}\left(e_{i_{1}}^{\left(a_{1}\right)} \cdots e_{i_{m}}^{\left(a_{m}\right)} f\right)(e) \cdot t_{1}^{a_{1}} \cdots t_{m}^{a_{m}} .
$$

(To prove (6.17), note that

$$
f\left(g x_{i}(t)\right)=\left(x_{i}(t) f\right)(g)=\left(\exp \left(t e_{i}\right) f\right)(g)=\sum_{a \geq 0} t^{a}\left(e_{i}^{(a)} f\right)(g) \quad(g \in G)
$$

and use induction on $m$.) If $f \in V_{\lambda}(\lambda-\beta)$ for some $\beta \in Q_{+}$then the tuples $\left(a_{1}, \ldots, a_{m}\right)$ that have a non-zero contribution to (6.17) must satisfy

$$
\sum_{k=1}^{m} a_{k} \alpha_{i_{k}}=\beta .
$$

In particular, for $f=\Delta^{\gamma}$ and $\mathbf{i} \in R(w)$, the only tuples that contribute to (6.17), must satisfy (6.18) with $\beta=\lambda-\gamma$. One of such tuples is the tuple $\left(b_{1}, \ldots, b_{m}\right)$ given by (6.13). Comparing (6.13) with (6.5) and remembering the normalization of $\Delta^{\gamma}$ given by (6.6), we see that the monomial $\prod_{k=1}^{m} t_{k}^{b_{k}}$ appears in $\Delta^{\gamma}$ with the coefficient 1 . The proof of (6.12) is completed by using the following lemma proved in $[1]$.

Lemma 6.5. In the situation of Lemma 6.4, if $a_{1}, \ldots, a_{m}$ are nonnegative integers satisfying (6.18) with $\beta=\lambda-\gamma$, and such that $e_{i_{1}}^{\left(a_{1}\right)} \cdots e_{i_{m}}^{\left(a_{m}\right)} \Delta^{\gamma} \neq 0$, then $\left(a_{1}, \ldots, a_{m}\right)$ coincides with the tuple $\left(b_{1}, \ldots, b_{m}\right)$ given by (6.13).

We have completed the proof of (6.9). To complete the proof of Theorem 6.2, it remains to show that

$$
M_{w_{k}^{\mathbf{i}} \omega_{i}}(x)=\Delta^{w_{k}^{\mathrm{i}} \omega_{i}}(y)
$$

for any $\mathbf{i}=\left(i_{1}, \ldots, i_{m}\right) \in R(w)$ and $k=1, \ldots, m$, where $x$ and $y$ are as in Theorem 6.2. (Note that (6.9) is a special case of (6.19) when $k=1$.) Let $u=$ $\left(w_{k}^{\mathbf{i}}\right)^{-1}=s_{i_{k}} \cdots s_{i_{m}}$, and let us write $x=x^{\prime \prime} x^{\prime}$, where $x^{\prime \prime}=x_{i_{1}}\left(t_{1}\right) \cdots x_{i_{k-1}}\left(t_{k-1}\right)$ 
and $x^{\prime}=x_{i_{k}}\left(t_{k}\right) \cdots x_{i_{m}}\left(t_{m}\right)$. By Proposition 5.5, the element $y$ can be written as $y=y^{\prime} y^{(k-1)} \cdots y^{(1)}$, where $y^{\prime} \in N(u), \eta_{u}\left(y^{\prime}\right)=x^{\prime}$, and each $y^{(l)}$ for $l<k$ belongs to the root subgroup $N\left(\alpha_{l}^{\mathbf{i}}\right)$. Since, in view of Proposition 2.1, none of the $\operatorname{roots} \alpha_{1}^{\mathbf{i}}, \ldots, \alpha_{k-1}^{\mathbf{i}}$ belong to $\operatorname{Inv}(u)$, it follows that the element $p=y^{(k-1)} \cdots y^{(1)}$ belongs to the subgroup $N^{\prime}(u)$ (see (5.2)). By Lemma 6.3 (applied to $u$ instead of $w)$

$$
\Delta^{w_{k}^{\mathbf{i}} \omega_{i}}(y)=\Delta^{u^{-1} \omega_{i}}\left(y^{\prime} p\right)=\Delta^{u^{-1} \omega_{i}}\left(y^{\prime}\right)
$$

On the other hand, it is clear from (4.13) that

$$
M_{w_{k}^{\mathbf{i}} \omega_{i}}(x)=M_{u^{-1} \omega_{i}}\left(x^{\prime}\right),
$$

where the right hand side of (6.21) is defined via the Chamber Ansatz for $u$ instead of $w$. Combining (6.20) and (6.21), we see that (6.19) becomes a consequence of (6.9), with $w, x$, and $y$ replaced by $u, x^{\prime}$, and $y^{\prime}$, respectively. Theorems 6.2 and 6.1, and hence Theorem 1.4, are proved.

As a first consequence of Theorem 6.1 , the relations between the $M_{\gamma}$ found in Section 4 (see Theorem 4.3 and Corollary 4.4) imply the corresponding relations between the functions $\Delta^{\gamma} \in \mathbf{C}\left[N_{-} \backslash G\right]$.

Corollary 6.6. The elements $M_{\gamma}=\Delta^{\gamma} \in \mathbf{C}\left[N_{-} \backslash G\right]$ satisfy the relations (4.5)(4.11).

Corollary 6.7. Let $\mathbf{i}=\left(i_{1}, \ldots, i_{m}\right) \in R(w)$, and let $\gamma$ be a w-chamber weight. Then, in the field of rational functions $\mathbf{C}\left(N_{-} \backslash G\right)$, the function $\Delta^{\gamma}$ can be expressed as a subtraction-free rational expression in the $\Delta^{\gamma(k ; \mathbf{i})}(k=1, \ldots, m)$.

Remark 6.8. It is well known that the $\operatorname{ring} \mathbf{C}\left[N_{-} \backslash G\right]$ is generated by the basis vectors in all fundamental representations $V_{\omega_{i}}$ (generalized Plücker coordinates) subject to quadratic Plücker relations. The functions $\Delta^{\gamma}$ corresponding to chamber weights $\gamma$ are the Plücker coordinates whose weights are vertices of weight polytopes for all $V_{\omega_{i}}$. It should be possible to deduce the relations $(4.6)-(4.11)$ from the Plücker relations, by eliminating all the coordinates whose weights lie inside the weight polytopes. For the type $A_{r}$, all the fundamental weights are minuscule (see [4], Ch. VIII, 7.3), i.e., all the weights for $V_{\omega_{i}}$ are extremal, so the $\Delta^{\gamma}$ form a complete set of Plücker coordinates. In general, this is not so, but the above results imply that the $\Delta^{\gamma}$ generate the field of fractions of $\mathbf{C}\left[N_{-} \backslash G\right]$.

Another consequence of Theorem 6.1 is Theorem 1.5 which produces a family of criteria for total positivity. This theorem can be restated as follows (cf. [2], Theorem 3.2.1).

Theorem 6.9. Let $x \in N^{w}=N \cap B_{-} w B_{-}$, and let $\mathbf{i}=\left(i_{1}, \ldots, i_{m}\right) \in R(w)$. Then the following are equivalent: 
(1) $x \in N_{>0}^{w}$;

(2) $\Delta^{\gamma}(x)>0$ for all $w$-chamber weights $\gamma$;

(3) $\Delta^{\gamma(k ; \mathbf{i})}(x)>0$ for $k=1, \ldots, m$.

Proof. The equivalence of (2) and (3) follows from Corollary 6.7. It remains to prove that $(1) \Leftrightarrow(3)$. The components $t_{1}, \ldots, t_{m}$ in the factorization $x=x_{\mathbf{i}}(t)=$ $x_{i_{1}}\left(t_{1}\right) \cdots x_{i_{m}}\left(t_{m}\right)$ and the functions $p_{1}, \ldots, p_{m}$ given by $p_{k}=\Delta^{\gamma(k ; \mathbf{i})}\left(\eta_{w}^{-1}(x)\right)$ form two systems of algebraic coordinates on $N^{w}$. By Proposition 1.1, the subset $N_{>0}^{w} \subset N^{w}$ can be defined by the inequalities $t_{1}>0, \ldots, t_{m}>0$. By Theorems 6.1 and 4.3 , the coordinates $\left(t_{1}, \ldots, t_{m}\right)$ and $\left(p_{1}, \ldots, p_{m}\right)$ are related to each other by an invertible monomial transformation (see also formulas (4.14) and (4.15) in the proof of Theorem 4.3). Thus, the positivity of all $t_{k}$ is equivalent to the positivity of all $p_{k}$. We conclude that $x \in N^{w}$ belongs to $N_{>0}^{w}$ if and only if $\Delta^{\gamma(k ; \mathbf{i})}(z)>0$ for $k=1, \ldots, m$, where $z=\eta_{w}^{-1}(x) \in N^{w}$. On the other hand, by Theorem 1.2 , $x \in N_{>0}^{w}$ if and only if $z \in N_{>0}^{w}$, and we are done.

We conclude this section with a generalization of some results of [7], which will allow us to produce more criteria for total positivity. Following Section 5 in [7], we will say that a collection $\left\{\gamma_{1}, \ldots, \gamma_{m}\right\}$ of $w$-chamber weights is a totally positive base for $N^{w}$ if for any $w$-chamber weight $\gamma$, the function $\Delta^{\gamma}$ can be expressed in the field of rational functions $\mathbf{C}\left(N_{-} \backslash G\right)$ as a subtraction-free rational expression in the $\Delta^{\gamma_{k}}(k=1, \ldots, m)$. In view of Theorem 6.9 , every such collection gives rise to a criterion for total positivity on $N^{w}$ : an element $x \in N^{w}$ belongs to $N_{>0}^{w}$ if and only if $\Delta^{\gamma_{k}}(x)>0$ for $k=1, \ldots, m$. For $\mathbf{i} \in R(w)$, let $\mathcal{C}(\mathbf{i})$ denote the collection $\{\gamma(1 ; \mathbf{i}), \ldots, \gamma(m ; \mathbf{i})\}$; by Corollary 6.7 , each $\mathcal{C}(\mathbf{i})$ is a totally positive base for $N^{w}$.

Let $i$ and $j$ be two different indices, and let $d$ be the order of $s_{i} s_{j}$ in $W$. If $d=3$, 4 , or 6 then by a weak $d$-flip we will mean the following operation on a collection $\mathcal{C}$ of chamber weights (cf [7], Section 5):

Case $d=3$. Replacing the weight $w^{\prime} s_{i} \omega_{i} \in \mathcal{C}$ with $w^{\prime} s_{j} \omega_{j}$, provided that $l\left(w^{\prime} s_{i} s_{j} s_{i}\right)=l\left(w^{\prime}\right)+3$, and that $\mathcal{C} \cup\left\{\omega_{i}, \omega_{j}\right\}$ contains four weights $w^{\prime} \omega_{i}, w^{\prime} \omega_{j}$, $w^{\prime} s_{j} s_{i} \omega_{i}$, and $w^{\prime} s_{i} s_{j} \omega_{j}$.

Case $d=4$. Replacing two weights $w^{\prime} s_{i} \omega_{i}$ and $w^{\prime} s_{i} s_{j} \omega_{j}$ in $\mathcal{C}$ with $w^{\prime} s_{j} \omega_{j}$ and $w^{\prime} s_{j} s_{i} \omega_{i}$, provided that $l\left(w^{\prime} s_{i} s_{j} s_{i} s_{j}\right)=l\left(w^{\prime}\right)+4$, and that $\mathcal{C} \cup\left\{\omega_{i}, \omega_{j}\right\}$ contains four weights $w^{\prime} \omega_{i}, w^{\prime} \omega_{j}, w^{\prime} s_{i} s_{j} s_{i} \omega_{i}$, and $w^{\prime} s_{j} s_{i} s_{j} \omega_{j}$.

Case $d=6$. Replacing four weights $w^{\prime} s_{i} \omega_{i}, w^{\prime} s_{i} s_{j} \omega_{j}, w^{\prime} s_{i} s_{j} s_{i} \omega_{i}$, and $w^{\prime} s_{i} s_{j} s_{i} s_{j} \omega_{j}$ in $\mathcal{C}$ with $w^{\prime} s_{j} \omega_{j}, w^{\prime} s_{j} s_{i} \omega_{i}, w^{\prime} s_{j} s_{i} s_{j} \omega_{j}$, and $w^{\prime} s_{j} s_{i} s_{j} s_{i} \omega_{i}$, provided that $l\left(w^{\prime} s_{i} s_{j} s_{i} s_{j} s_{i} s_{j}\right)=l\left(w^{\prime}\right)+6$, and that $\mathcal{C} \cup\left\{\omega_{i}, \omega_{j}\right\}$ contains four weights $w^{\prime} \omega_{i}$, $w^{\prime} \omega_{j}, w^{\prime} s_{i} s_{j} s_{i} s_{j} s_{i} \omega_{i}$, and $w^{\prime} s_{j} s_{i} s_{j} s_{i} s_{j} \omega_{j}$.

The following proposition is an immediate consequence of the relations (4.5)(4.11) (cf. [7], Proposition 5.10). 
Proposition 6.10. Weak d-flips preserve the set of all totally positive bases for $N^{w}$.

Note that if two reduced expressions $\mathbf{i}$ and $\mathbf{i}^{\prime}$ of $w$ are related to each other by a $d$-move then the corresponding totally positive bases $\mathcal{C}(\mathbf{i})$ and $\mathcal{C}\left(\mathbf{i}^{\prime}\right)$ are related to each other by a weak $d$-flip (if $d=2$ then $\mathcal{C}(\mathbf{i})=\mathcal{C}\left(\mathbf{i}^{\prime}\right)$ ). However, even when $\mathcal{C}=\mathcal{C}(\mathbf{i})$, it often happens that there exist weak $d$-flips on $\mathcal{C}$ that do not correspond to a $d$-move on $\mathbf{i}$. Such flips transform $\mathcal{C}(\mathbf{i})$ into a totally positive base which is not of the form $\mathcal{C}\left(\mathbf{i}^{\prime}\right)$ (a lot of examples of this kind for the type $A_{r}$ were given in [7]). It would be interesting to classify all totally positive bases obtained by applying weak $d$-flips to the bases of the form $\mathcal{C}(\mathbf{i})$.

\section{Special cases}

In this section we will give a graphical interpretation of the Chamber Ansatz for classical groups of types $A_{r}, B_{r}$, and $C_{r}$. We start by briefly recalling the $A_{r}$ case which was treated in [2] and served as a prototype for the results in the present paper. In this case $G=S L_{r+1}$, the maximal torus $H$ is the subgroup of diagonal matrices in $G$, and $N \subset G$ is the subgroup of unipotent upper-triangular matrices. The standard generators of $\mathbf{g}$ are:

$$
e_{i}=E_{i, i+1}, h_{i}=E_{i, i}-E_{i+1, i+1}, f_{i}=E_{i+1, i}(i=1, \ldots, r) .
$$

Thus, the one-parameter subgroups $x_{i}(t)$ in $N$ are given by $x_{i}(t)=1+t E_{i, i+1}$. The Weyl group $W$ is naturally identified with the symmetric group $S_{r+1}$, the simple reflection $s_{i}$ being identified with the transposition $(i, i+1)$. The anti-automorphisms $x \mapsto x^{T}$ and $x \mapsto x^{\iota}$ of $G$ act as follows: $x^{T}$ is the transpose of a matrix $x$, while $x^{\iota}=d_{0} x^{-1} d_{0}^{-1}$, where $d_{0}$ is a diagonal matrix $\operatorname{diag}\left(1,-1, \ldots,(-1)^{r}\right)$. Chamber weights of level $i$ are identified with the subsets of size $i$ in $[1, r+1]:=\{1, \ldots, r+1\}$ : the weight $\gamma$ corresponding to a subset $I$ is the character of $H$ given by

$$
d=\operatorname{diag}\left(d_{1}, \ldots, d_{r+1}\right) \mapsto d^{\gamma}=\prod_{i \in I} d_{i}
$$

Under this identification, the function $\Delta^{\gamma}(x)$ becomes the flag minor $\Delta^{I}(x)$, that is, the minor of $x$ with the row set $[1, i]$ and the column set $I$.

To visualize the Chamber Ansatz (see (1.10) or (4.4) above), we represent a reduced expression $\mathbf{i}=\left(i_{1}, \ldots, i_{m}\right) \in R(w)$ by means of its wiring diagram $\operatorname{Arr}(\mathbf{i})$. This diagram is a pseudo-line arrangement which can be drawn by combining segments taken from $r+1$ horizontal lines on the plane with $m \mathrm{X}$-shaped switches between them, where the $k$-th from the left switch occurs between the $i_{k}$-th and $\left(i_{k}+1\right)$-th lines, counting from the bottom. The pseudo-lines are labeled so that their right endpoints are numbered 1 through $r+1$ bottom-up; scanning the left 


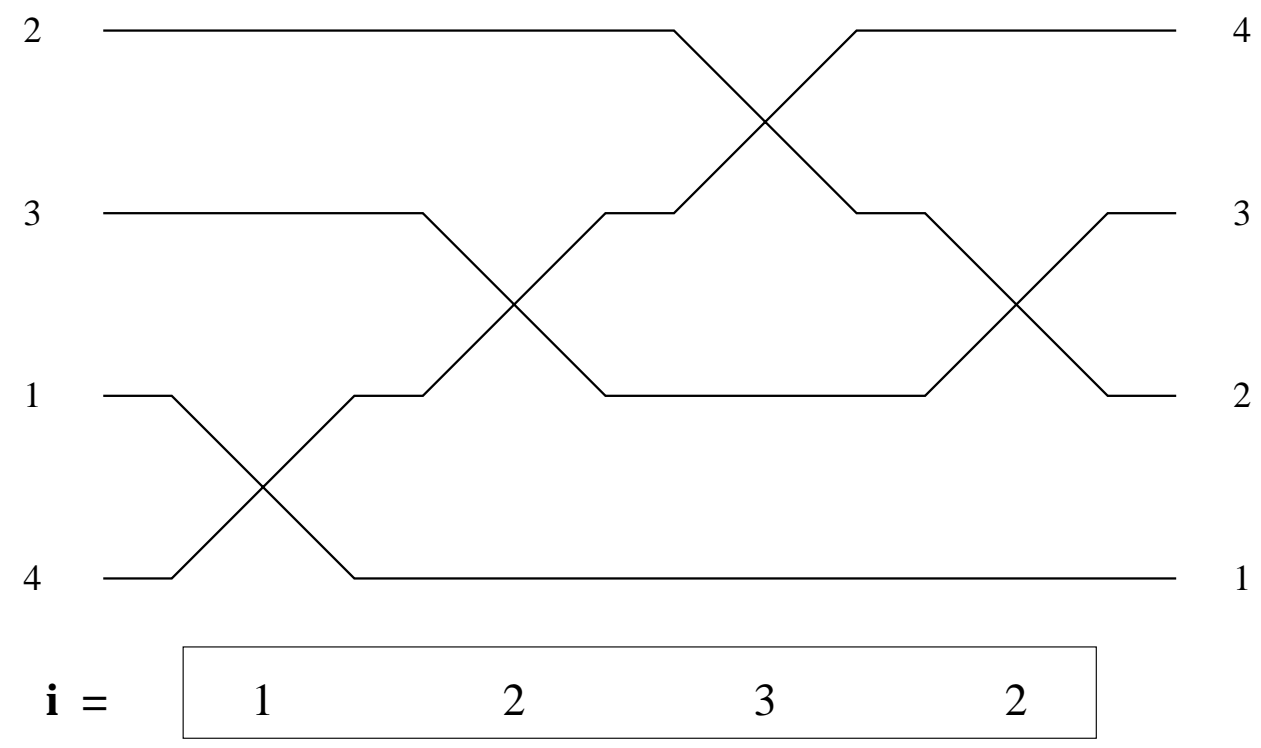

Figure 1

endpoints bottom-up yields the sequence $w^{-1}(1), \ldots, w^{-1}(r+1)$. See example in Figure 1, where $r=3$, and $\mathbf{i}=(1,2,3,2)$ (cf. [2], Figure 11). The coordinates $t_{1}^{\mathrm{i}}, \ldots, t_{m}^{\mathrm{i}}$ on the Lusztig variety $\mathcal{L}^{w}$ are associated with the crossings of pseudolines in Arr (i) scanning from the left end; we denote the $k$-th crossing from the left by the same symbol $t_{k}^{\mathbf{i}}$ as the corresponding coordinate.

The $\mathbf{i}$-chamber weights correspond to chambers of an arrangement Arr (i), that is, to the connected components of the complement of the union of all pseudo-lines. The chamber set associated to a chamber $C$ is the set of labels of all horizontal segments lying below $C$. The definitions readily imply that if $C$ is the chamber whose right end is $t_{k}^{\mathbf{i}}$, then the chamber set $L(C)$ corresponds to the chamber weight $\gamma(k ; \mathbf{i})$ (see $(2.10))$.

Using all this notation, Theorem 1.4 takes the following form: if $x=$ $x_{\mathbf{i}}\left(t_{1}, \ldots, t_{m}\right) \in N_{>0}^{w}$, and $z=\eta_{w}^{-1}(x)$ then

$$
t_{k}=\frac{\Delta^{L(A)}(z) \Delta^{L(D)}(z)}{\Delta^{L(B)}(z) \Delta^{L(C)}(z)},
$$

where $A, B, C$, and $D$ are the chambers of $\operatorname{Arr}(\mathbf{i})$ surrounding the crossing $t_{k}^{\mathbf{i}}$, with $A$ and $D$ lying above and below $t_{k}^{\mathrm{i}}$, and $B$ and $C$ being on the same horizontal level.

Our next target is the type $C_{r}$. The group $G$ is now the symplectic group $S p_{2 r}$. We will choose a matrix realization of $G$ most convenient for studying total 
positivity. Let $\tilde{G}=S L_{2 r}$, and consider two involutive anti-automorphisms $x \mapsto x^{\iota}$ and $x \mapsto x^{\tau}$ of $\tilde{G}$ given by

$$
x^{\iota}=d_{0} x^{-1} d_{0}^{-1}, x^{\tau}=w_{0} x^{T} w_{0}^{-1},
$$

where $d_{0}$ and $w_{0}$ are matrices of order $n=2 r$ given by

$$
\left(d_{0}\right)_{i j}=\delta_{i j}(-1)^{i-1},\left(w_{0}\right)_{i j}=\delta_{i, n+1-j} .
$$

The maps $x \mapsto x^{\iota}$ and $x \mapsto x^{\tau}$ commute with each other, so their composition is an involutive automorphism of $\tilde{G}$ given by

$$
x^{\iota \tau}=J^{-1}\left(x^{T}\right)^{-1} J,
$$

where $J=d_{0} w_{0}$ is the matrix given by

$$
J_{i j}=\delta_{i, n+1-j}(-1)^{i-1} .
$$

We will think of $G$ as the subgroup $\tilde{G}^{\iota \tau} \subset \tilde{G}$ of fixed points of the automorphism $\iota \tau$. In view of (7.6), an element $x \in \tilde{G}$ belongs to $G$ if and only if $x^{T} J x=J$; since $n=2 r$ is even, it follows from (7.7) that $J^{T}=-J$, so $G$ is indeed the symplectic group.

We will use our standard notation such as $N, W, x_{i}(t)$ etc., for the objects related to $G$, and will denote by $\tilde{N}, \tilde{W}, \tilde{x}_{i}(t)$ etc. the corresponding objects related to $\tilde{G}$. The antiautomorphisms $x \mapsto x^{\iota}$ and $x \mapsto x^{\tau}$ of $\tilde{G}$ preserve $\tilde{N}$ and $\tilde{H}$, and we have

$$
N=\tilde{N}^{\iota \tau}=\tilde{N} \cap G, H=\tilde{H}^{\iota \tau}=\tilde{H} \cap G .
$$

We set $i^{*}=n+1-i=2 r+1-i$ for $i=1, \ldots, 2 r$. In this notation, the standard generators of $\mathbf{g}$ are:

$$
\begin{gathered}
e_{i}=\tilde{e}_{i}+\tilde{e}_{i^{*}-1}, h_{i}=\tilde{h}_{i}+\tilde{h}_{i^{*}-1}, f_{i}=\tilde{f}_{i}+\tilde{f}_{i^{*}-1}(i=1, \ldots, r-1), \\
e_{r}=\tilde{e}_{r}, h_{r}=\tilde{h}_{r}, f_{r}=\tilde{f}_{r},
\end{gathered}
$$

where the $\tilde{e}_{i}, \tilde{h}_{i}$, and $\tilde{f}_{i}$ are given by (7.1). It follows that

$$
x_{i}(t)=\tilde{x}_{i}(t) \tilde{x}_{i^{*}-1}(t)(i=1, \ldots, r-1), x_{r}(t)=\tilde{x}_{r}(t) .
$$

The Weyl group $W$ of $G$ is naturally identified with the subgroup of $\tilde{W}=S_{n}=$ $S_{2 r}$ given by

$$
W=\left\{w \in S_{n}: w\left(i^{*}\right)=w(i)^{*}(i=1, \ldots, r)\right\} .
$$

Simple reflections in $W$ are expressed through simple reflections in $\tilde{W}$ as follows:

$$
s_{i}=\tilde{s}_{i} \tilde{s}_{i^{*}-1}(i=1, \ldots, r-1), s_{r}=\tilde{s}_{r} .
$$


Motivated by (7.12), we introduce the following notation: for a sequence $\mathbf{i}=$ $\left(i_{1}, \ldots, i_{m}\right)$ of indices taking values in $[1, r]$, we denote by $\tilde{\mathbf{i}}$ the sequence obtained from $\mathbf{i}$ by replacing each index $i_{k}=i<r$ with the pair $\left(i, i^{*}-1\right)$. For instance, if $r=2$ and $\mathbf{i}=(1,2,1,2)$ then $\tilde{\mathbf{i}}=(1,3,2,1,3,2)$. The following proposition is an easy consequence of (7.12) and (7.10).

\section{Proposition 7.1.}

(a) For any $w \in W$ and any reduced expression $\mathbf{i}=\left(i_{1}, \ldots, i_{m}\right)$ of $w$ in $W$, the sequence $\tilde{\mathbf{i}}$ is a reduced expression of $w$ in $\tilde{W}$.

(b) In the situation of (a), for any $t=\left(t_{1}, \ldots, t_{m}\right) \in \mathbf{C}^{m}$, we have

$$
x_{\mathbf{i}}(t)=\tilde{x}_{\tilde{\mathbf{i}}}(\tilde{t}),
$$

where $\tilde{t}$ is a sequence obtained from $t$ by replacing each $t_{k}$ such that $i_{k}<r$ with the pair $\left(t_{k}, t_{k}\right)$.

For instance, if $r=2$ and $\mathbf{i}=(1,2,1,2)$ then (7.13) takes the form

$$
x_{(1,2,1,2)}\left(t_{1}, t_{2}, t_{3}, t_{4}\right)=\tilde{x}_{(1,3,2,1,3,2)}\left(t_{1}, t_{1}, t_{2}, t_{3}, t_{3}, t_{4}\right) .
$$

As a corollary of Proposition 7.1, we can now describe a relationship between totally positive varieties in $N$ and $\tilde{N}$.

Corollary 7.2. For any $w \in W$, we have $N_{>0}^{w}=\tilde{N}_{>0}^{w} \cap N$. Therefore, $N_{\geq 0}=$ $\tilde{N}_{\geq 0} \cap N$.

Proof. The inclusion $N_{>0}^{w} \subset \tilde{N}_{>0}^{w} \cap N$ follows at once from (7.13) and Proposition 1.1. To prove the reverse inclusion, we notice that the automorphism $x \mapsto x^{\iota \tau}$ of $\tilde{G}$ sends $x_{i}(t)$ to $x_{i^{*}-1}(t)$, in particular, leaves $x_{r}(t)$ intact. Now let $\mathbf{i}=\left(i_{1}, \ldots, i_{m}\right)$ be a reduced expression of $w$ in $W$, and let $\mathbf{i}$ be the corresponding reduced expression of $w$ in $\tilde{W}$. Writing an element $x \in \tilde{N}_{>0}^{w}$ as the product $\tilde{x}_{\tilde{\mathbf{i}}}(p)$, where $p$ is some tuple of positive real numbers, and applying the automorphism $x \mapsto x^{\iota \tau}$ to this product, we conclude that $x^{\llcorner\tau}=x$ if and only if $p_{k}=p_{k+1}$ whenever $\tilde{i}_{k+1}=\tilde{i}_{k}^{*}-1$. Using (7.13) and Proposition 1.1 again, we see that if $x \in \tilde{N}_{>0}^{w}$ is such that $x^{\iota \tau}=x$ then $x$ belongs to $N_{>0}^{w}$, and we are done.

We now turn to the description of the Chamber Ansatz for $G$. First notice that the anti-automorphisms $x \mapsto x^{T}$ and $x \mapsto x^{\iota}$ of $G$ are obtained by restricting to $G$ the corresponding anti-automorphisms of $\tilde{G}$. Therefore, for any $w \in W$ the map $\eta_{w}$ for $G$ (see Proposition 5.1) agrees with the corresponding map for $\tilde{G}$. For $i=1, \ldots, r$, the fundamental weight $\omega_{i}$ for $G$ is the restriction to $H$ of the corresponding fundamental weight $\tilde{\omega}_{i}$ of $\tilde{G}$. The function $\Delta^{\omega_{i}}$ is the restriction to $G$ of $\Delta^{\tilde{\omega}_{i}}$, that is, $\Delta^{\omega_{i}}(x)=\Delta^{[1, i]}(x)$, the principal flag minor of $x \in G$. In view of (7.11), the correspondence $w^{-1} \omega_{i} \mapsto w^{-1}([1, i])$ identifies chamber weights of 


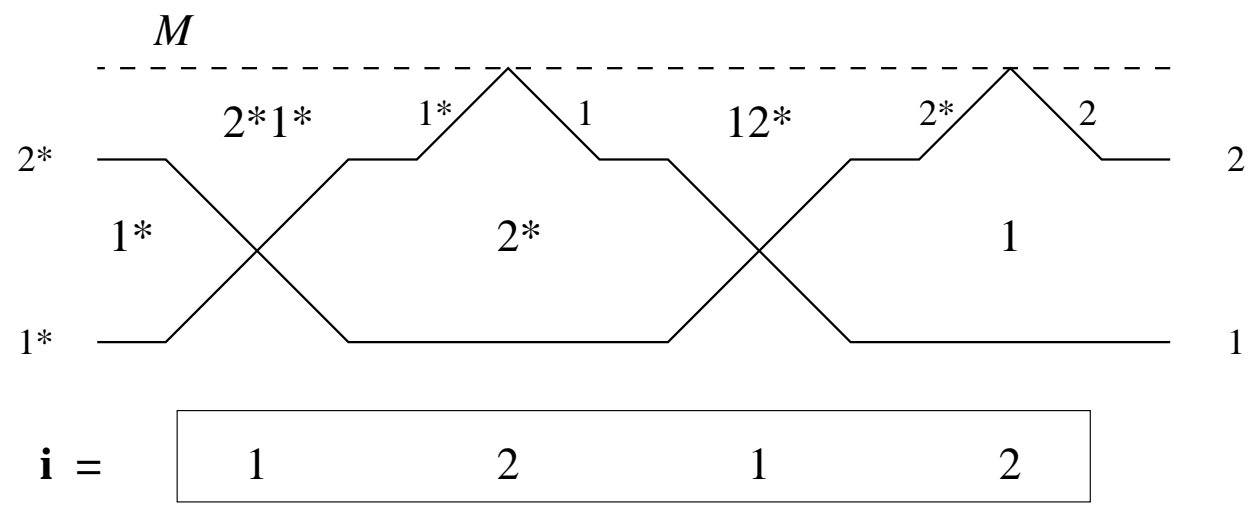

Figure 2

level $i$ for $G$ with isotropic subsets of size $i$ in $[1,2 r]$; here a subset $I \subset[1,2 r]$ is called isotropic if $I \cap I^{*}=\emptyset$, i.e., if $I$ contains at most one element from each pair $\left\{i, i^{*}\right\}$. Under this identification, the function $\Delta^{w^{-1} \omega_{i}}(x)$ becomes the flag minor $\Delta^{w^{-1}([1, i])}(x)$.

We will now introduce a wiring diagram for representing a reduced expression $\mathbf{i}=\left(i_{1}, \ldots, i_{m}\right)$ of $w \in W$ (see Figure 2 ). Let $\tilde{\mathbf{i}}$ be the corresponding reduced expression of $w$ in $\tilde{W}$ (see Proposition 7.1), and consider the pseudo-line arrangement $\operatorname{Arr}(\tilde{\mathbf{i}})$ representing $\tilde{\mathbf{i}}$. Each term $i_{k}=i<r$ in $\mathbf{i}$ creates a pair of consecutive crossings in Arr $(\tilde{\mathbf{i}})$, one on the level $i$ from the bottom and another on the level $i$ from the top. Since, for $i<r$, the simple reflections $\tilde{s}_{i}$ and $\tilde{s}_{i^{*}-1}$ in $\tilde{W}$ commute with each other (as well as the corresponding one-parameter subgroups $\tilde{x}_{i}(t)$ and $\tilde{x}_{i^{*}-1}(t)$ ), the order of these two consecutive crossings is irrelevant for applications such as the Chamber Ansatz; this makes it natural to put these two crossings on the same vertical line. With this convention, the arrangement $\operatorname{Arr}(\tilde{\mathbf{i}})$ has a horizontal symmetry axis that lies in the middle between the $r$-th and $(r+1)$ th horizontal lines. We denote this axis by $M$ (for "mirror"). Now we define the wiring diagram (or arrangement) $\operatorname{Arr}(\mathbf{i})$ as the half of the arrangement $\operatorname{Arr}(\tilde{\mathbf{i}})$ that lies below $M$. Thus, Arr (i) consists of $r$ pseudo-lines whose right endpoints are numbered 1 through $r$ bottom-up; scanning the left endpoints bottom-up yields the sequence $w^{-1}(1), \ldots, w^{-1}(r+1)$.

The crossings of $\operatorname{Arr}(\tilde{\mathbf{i}})$ that lie in $\operatorname{Arr}(\mathbf{i})$ will be called the crossings of $\operatorname{Arr}(\mathbf{i})$. They involve ordinary crossings, that is, the exchanges between the pairs of horizontal lines, and also the reflections in the "mirror" $M$. Under such a reflection, the pseudo-line changes its label from $i^{*}$ (to the left of the reflection point) to $i$, for some $i=1, \ldots, r$. By the definition, the arrangement $\operatorname{Arr}(i)$ has exactly $m=l(w)$ crossings. As in the case of $A_{r}$, we associate these crossings with the terms in the factorization $x_{\mathbf{i}}\left(t_{1}, \ldots, t_{m}\right)$; we will again denote a crossing by the same symbol $t_{k}=t_{k}^{\mathrm{i}}$ as the corresponding coordinate on the Lusztig variety. 
The chambers and chamber sets for Arr (i) are defined in the same way as for $A_{r}$. In full analogy with the $A_{r}$ case, if $C$ is the chamber whose right end is $t_{k}^{\mathbf{i}}$ then the chamber set $L(C)$ is the isotropic subset corresponding to the chamber weight $\gamma(k ; \mathbf{i})$ (see $(2.10))$.

Now everything is ready for a "concrete" formulation of Theorem 1.4 for the type $C_{r}$. Let $x=x_{\mathbf{i}}\left(t_{1}, \ldots, t_{m}\right) \in N_{>0}^{w}$, and let $z=\eta_{w}^{-1}(x)$. If $i_{k}<r$ then the crossing $t_{k}$ in $\operatorname{Arr}(\mathbf{i})$ is surrounded by four chambers $A, B, C$, and $D$ as in the case $A_{r}$, and $t_{k}$ is given by (7.3). If $i_{k}=r$, i.e., the corresponding crossing $t_{k}$ lies on the mirror $M$ then this crossing is surrounded by three chambers: the chamber $A$ below $t_{k}$, and the chambers $B$ and $C$ just below the mirror on both sides of $t_{k}$. Then (1.10) takes the following form:

$$
t_{k}=\frac{\left(\Delta^{L(A)}(z)\right)^{2}}{\Delta^{L(B)}(z) \Delta^{L(C)}(z)}
$$

Note that (7.14) can be deduced from the Chamber Ansatz for type $A_{n-1}$ with the help of the following lemma.

Lemma 7.3. For any subset $I \subset[1, n]=[1,2 r]$ and any $x \in G$, we have $\Delta^{I}(x)=$ $\Delta^{\bar{I}^{*}}(x)$, where $\bar{I}$ is the complement of $I$ in $[1, n]$.

This lemma follows from the observation that $\Delta^{I}\left(x^{\iota \tau}\right)=\Delta^{\bar{I}^{*}}(x)$ for any $x \in \tilde{G}$ (cf. [2], (3.4.4)).

Applying Lemma 7.3 to a subset $L(A)$ in (7.14) (which is an isotropic subset of size $r-1)$, we conclude that $\Delta^{L(A)}(z)=\Delta^{L(D)}(z)$, where $D$ is the chamber above $t_{k}$ in $\operatorname{Arr}(\tilde{\mathbf{i}})$. Therefore, (7.14) becomes a consequence of (7.13) and (7.3), the latter applied to the reduced expression $\tilde{\mathbf{i}}$.

We now turn to the type $B_{r}$. The group $G$ is the spin group $\operatorname{Spin}_{2 r+1}$ which is a two-sheeted covering over the special orthogonal group $G_{0}=S O_{2 r+1}$. Since the projection $G \rightarrow G_{0}$ restricts to an isomorphism of the maximal unipotent subgroup $N$ of $G$ with that of $G_{0}$, in studying the totally positive varieties in $N$ we can (and will) work with $G_{0}$ rather than with $G$. Our treatment of $G_{0}$ will be completely parallel to the above treatment of the symplectic group. We set $n=2 r+1$ and $\tilde{G}=S L_{n}$, and we think of $G_{0}$ as the subgroup $\tilde{G}^{i \tau} \subset \tilde{G}$ of fixed points of the automorphism $\iota \tau$, where the involutive anti-automorphisms $x \mapsto x^{\iota}$ and $x \mapsto x^{\tau}$ of $\tilde{G}$ are given by (7.4) and (7.5). Formulas (7.6) and (7.7) remain valid; the only difference with the symplectic case is that now the matrix $J$ given by (7.7) is symmetric, so $G_{0}$ is indeed the special orthogonal group. With the same notational conventions as for the symplectic group, (7.8) also remains valid in our case.

We set $i^{*}=n+1-i=2 r+2-i$ for $i=1, \ldots, 2 r+1$. In this notation, the 
standard generators of $\mathbf{g}$ are:

$$
\begin{array}{ll}
e_{i}=\tilde{e}_{i}+\tilde{e}_{i^{*}-1}, & h_{i}=\tilde{h}_{i}+\tilde{h}_{i^{*}-1}, \quad f_{i}=\tilde{f}_{i}+\tilde{f}_{i^{*}-1}(i=1, \ldots, r-1), \\
e_{r}=\sqrt{2}\left(\tilde{e}_{r}+\tilde{e}_{r+1}\right), h_{r}=2\left(\tilde{h}_{r}+\tilde{h}_{r+1}\right), f_{r}=\sqrt{2}\left(\tilde{f}_{r}+\tilde{f}_{r+1}\right),
\end{array}
$$

where the $\tilde{e}_{i}, \tilde{h}_{i}$, and $\tilde{f}_{i}$ are given by (7.1). (The coefficients $\sqrt{2}$ in $(7.15)$ are necessary if we want the anti-automorphism $x \mapsto x^{T}$ of $G_{0}$ to be the ordinary transpose of matrices.) The analogue of (7.10) is now

$$
x_{i}(t)=\tilde{x}_{i}(t) \tilde{x}_{i^{*}-1}(t)(i=1, \ldots, r-1), x_{r}(t)=\tilde{x}_{r}\left(\frac{t}{\sqrt{2}}\right) \tilde{x}_{r+1}(\sqrt{2} t) \tilde{x}_{r}\left(\frac{t}{\sqrt{2}}\right)
$$

(the last equality in (7.16) is proved by a direct calculation in $S L_{3}$ ).

The Weyl group $W$ of $G$ is naturally identified with the subgroup of $\tilde{W}=S_{n}=$ $S_{2 r+1}$ given by (7.11). Since $n$ is now odd, all permutations from $W$ leave $r+1$ fixed; restricting these permutations to $[1, n]-\{r+1\}=[1, r] \cup[1, r]^{*}$ we obtain a natural isomorphism of $W$ with the Weyl group for the type $C_{r}$. Simple reflections in $W$ are expressed through simple reflections in $\tilde{W}$ as follows:

$$
s_{i}=\tilde{s}_{i} \tilde{s}_{i^{*}-1}(i=1, \ldots, r-1), s_{r}=\tilde{s}_{r} \tilde{s}_{r+1} \tilde{s}_{r} .
$$

Modifying the corresponding construction for the type $C_{r}$, for a sequence $\mathbf{i}=$ $\left(i_{1}, \ldots, i_{m}\right)$ of indices taking values in $[1, r]$, we denote by $\tilde{\mathbf{i}}$ the sequence obtained from $\mathbf{i}$ by replacing each index $i_{k}=i<r$ with the pair $\left(i, i^{*}-1\right)$, and each index $i_{k}=r$ with the triple $(r, r+1, r)$. For instance, if $r=2$ and $\mathbf{i}=(1,2,1,2)$ then $\tilde{\mathbf{i}}=$ $(1,4,2,3,2,1,4,2,3,2)$. We then have the following analogue of Proposition 7.1.

\section{Proposition 7.4.}

(a) For any $\underset{\tilde{\mathbf{i}}}{w} \in W$ and any reduced expression $\mathbf{i}=\left(i_{1}, \ldots, i_{m}\right)$ of $w$ in $W$, the sequence $\tilde{\mathbf{i}}$ is a reduced expression of $w$ in $\tilde{W}$.

(b) In the situation of (a), for any $t=\left(t_{1}, \ldots, t_{m}\right) \in \mathbf{C}^{m}$, we have

$$
x_{\mathbf{i}}(t)=\tilde{x}_{\tilde{\mathbf{i}}}(\tilde{t}),
$$

where $\tilde{t}$ is a sequence obtained from $t$ by replacing each $t_{k}$ such that $i_{k}<r$ with the pair $\left(t_{k}, t_{k}\right)$ and each $t_{k}$ such that $i_{k}=r$ with the triple $\left(\frac{t_{k}}{\sqrt{2}}, \sqrt{2} t_{k}, \frac{t_{k}}{\sqrt{2}}\right)$.

For instance, if $r=2$ and $\mathbf{i}=(1,2)$ then (7.18) takes the form

$$
x_{(1,2)}\left(t_{1}, t_{2}\right)=\tilde{x}_{(1,4,2,3,2)}\left(t_{1}, t_{1}, \frac{t_{2}}{\sqrt{2}}, \sqrt{2} t_{2}, \frac{t_{2}}{\sqrt{2}}\right) .
$$

As in the case of $C_{r}$, we conclude that Corollary 7.2 remains valid for the type $B_{r}$. The same proof applies, with the following modification. We need to show 
that an element $x=\tilde{x}_{r}\left(p_{1}\right) \tilde{x}_{r+1}\left(p_{2}\right) \tilde{x}_{r}\left(p_{3}\right)$ with $p_{1}, p_{2}, p_{3}>0$ is invariant under the automorphism $x \mapsto x^{\iota \tau}$ if and only if $p_{2}=2 p_{1}=2 p_{3}$, i.e., $\left(p_{1}, p_{2}, p_{3}\right)=$ $\left(\frac{t}{\sqrt{2}}, \sqrt{2} t, \frac{t}{\sqrt{2}}\right)$ for some $t>0$. Using the transition rule (3.3), we see that

$$
x^{\iota \tau}=\tilde{x}_{r+1}\left(p_{1}\right) \tilde{x}_{r}\left(p_{2}\right) \tilde{x}_{r+1}\left(p_{3}\right)=\tilde{x}_{r}\left(\frac{p_{2} p_{3}}{p_{1}+p_{3}}\right) \tilde{x}_{r+1}\left(p_{1}+p_{3}\right) \tilde{x}_{r}\left(\frac{p_{1} p_{2}}{p_{1}+p_{3}}\right) .
$$

Therefore, the condition that $x^{\iota \tau}=x$ is equivalent to $\left(p_{1}, p_{2}, p_{3}\right)=\left(\frac{p_{2} p_{3}}{p_{1}+p_{3}}, p_{1}+\right.$ $\left.p_{3}, \frac{p_{1} p_{2}}{p_{1}+p_{3}}\right)$ which is easily seen to be equivalent to $p_{2}=2 p_{1}=2 p_{3}$.

As in the case of $C_{r}$, the anti-automorphisms $x \mapsto x^{T}$ and $x \mapsto x^{\iota}$ of $G_{0}$, as well as the map $\eta_{w}$ for any $w \in W$, agree with the corresponding maps for $\tilde{G}$. For $i=1, \ldots, r-1$, the descriptions of the fundamental weight $\omega_{i}$, the chamber weights of level $i$ and the corresponding functions $\Delta^{\gamma}$ are the same as for the type $C_{r}$. Thus, chamber weights of level $i<r$ are identified with isotropic subsets of size $i$ in $[1, r] \cup[1, r]^{*}=[1,2 r+1]-\{r+1\}$; under this identification, the function $\Delta^{w^{-1} \omega_{i}}(x)$ on $G_{0}$ becomes the flag minor $\Delta^{w^{-1}([1, i])}(x)$.

The situation with level $r$ is somewhat different. The fundamental weight $\omega_{r}$ is the highest weight of the spinor representation of $G$; thus, the restriction to $H$ of the fundamental weight $\tilde{\omega}_{r}$ is the square of the character $\omega_{r}$ (see, e.g., [5], Ch. 20). It follows that chamber weights of level $r$ are still identified with isotropic subsets of size $r$ in $[1, r] \cup[1, r]^{*}=[1,2 r+1]-\{r+1\}$; however, the function $\Delta^{w^{-1} \omega_{r}}(x)$ is now identified with $\sqrt{\Delta^{w^{-1}([1, r])}}(x)$, the square root of the corresponding flag minor. Note that $\sqrt{\Delta^{w^{-1}([1, r])}}$ is not a well-defined function on the whole group $G_{0}=S O_{2 r+1}$, only on its two-sheeted covering $G=S$ pin $_{2 r+1}$; but its restriction to $N$ is a well-defined regular function, since $N$ is identified with the maximal unipotent subgroup of $G$. We only have to specify the choice of the sign of the square root. This can be done by using the normalization condition (6.6). To be more precise, we note that the spinor representation $V_{\omega_{r}}$ of $G$ is minuscule (cf. Remark 6.8). Thus, its set of weights is the set $W \omega_{r}$ of chamber weights of level $r$, so these weights are identified with isotropic subsets $I \subset[1, r] \cup[1, r]^{*}$ of size $r$. The corresponding functions $\sqrt{\Delta^{I}}$ form a basis in $V_{\omega_{r}}$. The action of the raising operators $e_{1}, \ldots, e_{r}$ on this basis can be described as follows (cf. [2], (3.5.8)): for $i=1, \ldots, r-1$ we have

$$
e_{i}\left(\sqrt{\Delta^{I}}\right)= \begin{cases}\sqrt{\Delta^{I \cup\left\{i, i^{*}-1\right\}-\left\{i+1, i^{*}\right\}}} & , \text { if } I \cap\left\{i, i+1, i^{*}-1, i^{*}\right\}=\left\{i+1, i^{*}\right\} \\ 0 & , \text { otherwise; }\end{cases}
$$

the action of $e_{r}$ is given by

$$
e_{r}\left(\sqrt{\Delta^{I}}\right)= \begin{cases}\sqrt{\Delta^{I \cup\{r\}-\left\{r^{*}\right\}}} & , \text { if } I \cap\left\{r, r^{*}\right\}=\left\{r^{*}\right\} \\ 0 & , \text { otherwise. }\end{cases}
$$

Formulas (7.19) and (7.20) imply, in particular, that all operators $e_{i}^{2}$ acting on $V_{\omega_{r}}$ are equal to 0 (this is a general property of minuscule representations). 
Combining this fact with (6.17), we can obtain an explicit combinatorial expression for $\sqrt{\Delta^{I}}$ similar to [2], (2.4.8). To do this, consider the vector space of formal linear combinations of isotropic subsets of size $r$ in $[1, r] \cup[1, r]^{*}$. Define the shift operators $u_{1}, \ldots, u_{r}$ in this space by setting

$$
u_{i}(I)= \begin{cases}I \cup\left\{i, i^{*}-1\right\}-\left\{i+1, i^{*}\right\} & , \text { if } \quad I \cap\left\{i, i+1, i^{*}-1, i^{*}\right\}=\left\{i+1, i^{*}\right\} \\ 0 & , \text { otherwise }\end{cases}
$$

for $i<r$, and

$$
u_{r}(I)= \begin{cases}I \cup\{r\}-\left\{r^{*}\right\} & , \text { if } \quad I \cap\left\{r, r^{*}\right\}=\left\{r^{*}\right\} \\ 0 & , \text { otherwise. }\end{cases}
$$

As a consequence of $(6.17)$, for any sequence $\mathbf{i}=\left(i_{1}, \ldots, i_{m}\right)$ of indices from $[1, r]$, and any complex numbers $t_{1}, \ldots, t_{m}$, we have

$$
\sqrt{\Delta^{I}}\left(x_{\mathbf{i}}\left(t_{1}, \ldots, t_{m}\right)\right)=\sum t_{a_{1}} t_{a_{2}} \cdots t_{a_{s}},
$$

where the sum is over all sequences $1 \leq a_{1}<a_{2}<\cdots<a_{s} \leq m$ such that

$$
u_{i_{a_{1}}} u_{i_{a_{2}}} \cdots u_{i_{a_{s}}}(I)=[1, r]
$$

For example, if $r=2$ and $x=x_{(1,2,1,2)}\left(t_{1}, t_{2}, t_{3}, t_{4}\right)$ then $(7.23)$ gives

$$
\begin{gathered}
\sqrt{\Delta^{\{1,2\}}}(x)=1, \quad \sqrt{\Delta^{\left\{1,2^{*}\right\}}}(x)=t_{2}+t_{4}, \\
\sqrt{\Delta^{\left\{2,1^{*}\right\}}}(x)=t_{2} t_{3}, \quad \sqrt{\Delta^{\left\{2^{*}, 1^{*}\right\}}}(x)=t_{2} t_{3} t_{4} .
\end{gathered}
$$

Returning to the Chamber Ansatz, we recall that the Weyl group $W$ for the type $B_{r}$ is canonically identified with the Weyl group for the type $C_{r}$, by means of the order-preserving bijection $[1, r] \cup[1, r]^{*}=[1,2 r+1]-\{r+1\} \rightarrow[1,2 r]$. Using this identification, for any $w \in W$ and any reduced expression $\mathbf{i} \in R(w)$ in the $B_{r}$ case, we define the pseudo-line arrangement Arr (i), its chambers and chamber sets in exactly the same way as for the type $C_{r}$ above (see Figure 2). So the only difference between two cases is that whenever we see an index $i^{*}$ for some $i \in[1, r]$, either as the label of a segment of a pseudo-line, or as a member of a chamber set, it is understood as $2 r+2-i$ in the $B_{r}$ case, and as $2 r+1-i$ in the $C_{r}$ case.

We are now in a position to give a "concrete" formulation of Theorem 1.4 for the type $B_{r}$. Let $x=x_{\mathbf{i}}\left(t_{1}, \ldots, t_{m}\right) \in N_{>0}^{w}$, and let $z=\eta_{w}^{-1}(x)$. If $i_{k}<r$ then the crossing $t_{k}$ in $\operatorname{Arr}(\mathbf{i})$ is surrounded by four chambers $A, B, C$, and $D$ as in the cases $A_{r}$ or $C_{r}$ above, and $t_{k}$ is given by (7.3). If $i_{k}=r$, i.e., the corresponding crossing $t_{k}$ lies on the mirror $M$ then this crossing is surrounded by three chambers: the 
chamber $A$ below $t_{k}$, and the chambers $B$ and $C$ just below the mirror on both sides of $t_{k}$. Then (1.10) takes the following form (cf. (7.14)):

$$
t_{k}=\frac{\Delta^{L(A)}(z)}{\sqrt{\Delta^{L(B)}}(z) \sqrt{\Delta^{L(C)}}(z)} .
$$

Note that Lemma 7.3 and its proof remain valid for the type $B_{r}$. Using this lemma and comparing (7.24) with the expression for $\sqrt{2} t_{k}$ given by the Chamber Ansatz for the type $A_{2 r}$ applied to the right hand side of (7.18), we arrive at the following identity.

Proposition 7.5. Let $L \subset[1, r] \cup[1, r]^{*}$ be an isotropic subset of size $r-1$, and let $i \in[1, r]$ be the index such that $L \cap\left\{i, i^{*}\right\}=\emptyset$. Then

$$
\Delta^{L \cup\{r+1\}}(x)=\sqrt{2} \sqrt{\Delta^{L \cup\{i\}}}(x) \sqrt{\Delta^{L \cup\left\{i^{*}\right\}}}(x)
$$

for any $x \in G_{0}=S O_{2 r+1}$.

Remark 7.6. The realization of the symplectic group $S p_{2 r}$ as a subgroup of $S L_{2 r}$ that we used above, agrees with the general strategy of Lusztig using the "descent" realization of a non-simply-laced semisimple group as a subgroup of a simply laced one. In particular, Corollary 7.2 for the type $C_{r}$ is a special case of a general result by Lusztig. However, the above realization of $S O_{2 r+1}$ as a subgroup of $S L_{2 r+1}$ does not follow this pattern; in Lusztig's approach, $S O_{2 r+1}$ would be realized as a subgroup of the group $S O_{2 r+2}$ of type $D_{r+1}$. It is possible to treat the case $D_{r}$ by using an embedding of $S O_{2 r}$ into $S L_{2 r}$. However, the resulting description of totally positive varieties is not as nice as for the types $B_{r}$ and $C_{r}$ (in particular, there seems to be no way to satisfy Corollary 7.2), and we do not give it here.

\section{References}

[1] A. Berenstein, Group-like elements in quantum groups and Feigin's conjecture, 1996.

[2] A. Berenstein, S. Fomin and A. Zelevinsky, Parametrizations of canonical bases and totally positive matrices, Adv. in Math. 122 (1996), 49-149.

[3] N. Bourbaki, Groupes et algèbres de Lie, Ch. IV-VI, Hermann, Paris 1968.

[4] N. Bourbaki, Groupes et algèbres de Lie, Ch. VII-VIII, Hermann, Paris 1975.

[5] W. Fulton and J. Harris, Representation theory, Springer-Verlag, New York 1991.

[6] J. E. Humphreys, Reflection groups and Coxeter groups, Cambridge University Press, Cambridge 1994.

[7] B. Leclerc and A. Zelevinsky, Quasicommuting families of quantum Plücker coordinates, preprint, Université de Caen, Caen 1996.

[8] G. Lusztig, Introduction to quantized enveloping algebras. In: J. Tirao, N. Wallach (Eds.), Progr. in Math. 105, Birkhäuser, Boston 1992, 49-65.

[9] G. Lusztig, Introduction to quantum groups, Birkhäuser, Boston 1993. 
[10] G. Lusztig, Total positivity in reductive groups, in: Lie theory and geometry: in honor of Bertram Kostant, Progress in Mathematics 123, Birkhäuser, Boston 1994.

[11] G. Lusztig, Total positivity and canonical bases, preprint.

Arkady Berenstein

Department of Mathematics

Cornell University

Ithaca, NY 14853

USA

e-mail: arkady@math.cornell.edu
Andrei Zelevinsky

Department of Mathematics

Northeastern University

Boston, MA 02115

USA

e-mail: andrei@neu.edu

(Received: October 3, 1996) 